\title{
Leseleistung und Rezeptionsstrategien bei Mädchen und Jungen in der Sekundarstufe I
}

\author{
Doris Grütz (Weingarten)
}

\begin{abstract}
Do girls read texts differently from boys? This contribution presents a study from empirical teaching research in secondary education ("Sekundarstufe 1") by Grütz/Belgrad/Pfaff (2005). 4500 pupils, male and female, from the $7^{\text {th }}$ form of primary and secondary school were examined to determine which of the reading strategies usually adopted lead to a more successful text understanding with girls and which with boys. This study follows up a previous study done in $4^{\text {th }}$ form primary school by Belgrad/Grütz/Pfaff (2003). The research design is based on the psychological cognition model of text understanding following van Dijk/Kintsch (1983), which sees the process of reading comprehension as interactional and constructive. Cognitive reading strategies were tested which are usually adopted during lessons. Major results of the study are that boys prefer similar reading strategies both in primary and secondary schools. Good results are achieved when a text is read quietly and discussed afterwards. The worst results are obtained by all boys when words have to be copied from the text. With girls a notable difference can be seen in the usage of reading strategies depending on the type of the school and the level of reading competence. Girls from primary schools achieve the best results when they can activate knowledge prior to confronting the text, girls from secondary schools achieve the best results when they quietly read through a text.
\end{abstract}

\section{$1 \quad$ Beschreibung der Studie}

Im vorliegenden Beitrag geht es um die geschlechtsspezifischen Ergebnisse der Studie zu Rezeptionsstrategien von Sachtexten in der 7. Klasse Haupt- und Realschule von Grütz/Belgrad/Pfaff (2004). Es wird folgenden zwei Fragen nachgegangen: Erstens: Unterscheiden sich die Leseleistungen von Mädchen und Jungen in der Sekundarstufe I ? D.h., ist ein Geschlecht beim Rezipieren von Sachtexten dem andern überlegen? Zweitens: Werden von Mädchen und Jungen unterschiedliche Rezeptionsstrategien bevorzugt? Diese Studie ist eine Anschlussstudie an eine Untersuchung in der 4. Klasse Grundschule mit gleichem Forschungsdesign (Belgrad/Grütz/Pfaff 2003), deren genderspezifische Ergebnisse in einem früheren Heft dargestellt wurden (Grütz 2004). ${ }^{1}$

1 In beiden Studien wurde neben der Frage nach geschlechtsspezifischen Leseleistungen und Rezeptionsstrategien auch folgenden Fragen nachgegangen: Welche Leseleistungen und bevorzugte 


\subsection{Leseverstehenstheoretische Grundlagen}

Theoretische Grundlage für die ausgewählten Strategien zur Rezeption von Sachtexten bildet das Situationsmodell von Kintsch/van Dijk (1983). Textverstehen wird als Prozess interaktiven Konstruierens von Bedeutung verstanden, bei dem Hypothesen aufgestellt und überprüft sowie aufgrund des Vor- und Weltwissens Inferenzen gezogen werden. Zwei Komponenten wirken beim Rezipieren zusammen: der Aufbau einer propositionalen Textbasis auf der Grundlage des Textes (Bottom-up-Prozesse) und der Aufbau eines Situationsmodells aufgrund leserseitiger Dispositionen (Top-down-Prozesse). Zu den das Situationsmodell generierenden Faktoren, die der Leser/die Leserin als kognitive, motivationale und emotionale Voraussetzungen mitbringt, gehören Vorwissen (textbezogene Ereignisse, Handlungen), Weltwissen, Sprachwissen, Leseziele und ebenso die pragmatische, interaktive und situative Einbettung des Textes. Aufgrund des Zusammenspiels mehrerer Komponenten des Textverstehens legt das Modell eine kognitive Flexibilität der Rezipierenden nahe. ${ }^{2}$ Von der kognitiven Flexibilität des Rezipierenden hängt auch der von uns verwendete Strategiebegriff ab. "Strategie" suggeriert zunächst ein bewusstes Anwenden einer bestimmten Methode (Paris et al. 1986). Man geht in der neueren Leseforschung allerdings davon aus, dass eine kompetente Leserin bzw. Leser eine Vielzahl von Dekodierund Verstehensverfahren automatisiert hat (Pressley et al. 1985; Kintsch 1996) und nur im Falle eines Verstehenshindernisses - aufgrund metakognitiver Kompetenz - bewusst Strategien einsetzt (Artelt, 2004: 61f). Strategien zum Textverstehen werden hier mit Artelt verstanden als "zielgerichtete, potenziell bewusste und kontrollierbare Prozesse" (ebd.: 62).

\subsection{Forschungsdesign}

Um der Frage nachzugehen, welche Rezeptionsstrategien am ehesten geeignet sind, Sachtexte zu verstehen und ihnen Informationen zu entnehmen, flossen obige Überlegungen zur Theorie des Leseverstehens ein. Es sollte zudem eine typische schulische Rezeptionssituation reflektiert und in das Forschungsdesign integriert werden. Nach Auskunft der Lehrkräfte werden im Unterricht in der Regel zwei Rezeptionsdurchgänge durchgeführt: Der erste muss für die Textbegegnung geeignet sein, der zweite soll dem vertieften Rezipieren dienen. Das zweimalige Rezipieren entspricht auch Empfehlungen der experimentellen Lesetrainingsforschung, die die Kombination unterschiedlicher Strategien anstelle von Einzelstrategien nahe legen (Friedrich 1995: 23ff.). Für die beiden Rezeptionsdurchgänge boten sich folgende Methoden $a^{3}$ :

\footnotetext{
Rezeptionsstrategien zeigen Schüler/innen in der Haupt- und Realschule? Welche Unterschiede bestehen zwischen Schüler/innen deutscher Erstsprache und Schüler/innen mit Migrationshintergrund.

2 Die ausführliche Diskussion leseverstehenstheoretischer Modelle als Grundlage für die Rezeptionsstrategien findet sich in Belgrad/Grütz/Pfaff (2003): Forschungsbericht zur Studie in der Grundschule sowie in Grütz/Belgrad/Pfaff (2004): Forschungsbericht zur Studie in der Sekundarstufe 1. www.ph-weingarten.de/deutsch.

${ }^{3}$ Zur ausführlichen kognitionspsychologischen Begründung der einzelnen Methoden: ebda.
} 


\section{Rezeptionsdurchgang:}

1. Stillesen (Die Schüler/innen lesen den Text still durch.)

2. Vorlesen (Die Lehrkraft liest den Text laut vor.)

3. Aktivieren von Vorwissen im Unterrichtsgespräch (Leitfragen seitens der Lehrkraft lassen eine Erwartungshaltung der Schüler/innen an den Text entstehen.)

\section{Rezeptionsdurchgang}

1. Unterstreichen (Der Text wird still gelesen und dabei werden ca. zehn Wörter unterstrichen.)

2. Reden (Der Text wird still gelesen und danach wird über den Textinhalt gesprochen.)

3. Schreiben

(Der Text wird still gelesen und dabei werden ca. zehn Wörter herausgeschrieben.)

Bei den Rezeptionsstrategien handelt es sich um unterschiedliche Arten von Lesestrategien: Als Lesestrategien im engeren Sinn werden das stille Lesen im ersten Rezeptionsdurchgang und die reduktiv-organisatorischen Strategien Unterstreichen und Herausschreiben von Schlüsselwörtern im zweiten Rezeptionsdurchgang verstanden. Wenn es hingegen im ersten Rezeptionsdurchgang darum geht, Vorwissen im Unterrichtsgespräch zu aktivieren, und im zweiten Rezeptionsdurchgang darum, im Unterrichtsgespräch über das Thema des Textes zu reden, handelt es sich um Strategien, die das Leseverstehen vorbereiten und elaborativ nachbereiten. Diese können als Lesestrategien i. w. S. oder als Lesestützstrategien bezeichnet werden. Beim Vorlesen des Textes durch die Lehrkraft handelt es sich gar um eine Hörstrategie. Im Sinne einer begrifflichen Einheitlichkeit entschieden wir uns für den weiter gefassten Begriff "Rezeptionsstrategien".

Die Methoden des ersten und des zweiten Rezeptionsdurchgangs wurden miteinander kombiniert, so dass es neun Kombinationen von Rezeptionsmethoden ergab. Sie sind im Modell durch neun Felder (M 1 - 9) abgebildet (Abb. 1).

\begin{tabular}{|c|c|c|c|}
\hline & M 1 & M 2 & M 3 \\
\hline $\begin{array}{c}\text { 1. Rezeptions- } \\
\text { durchgang }\end{array}$ & Still Lesen & Still Lesen & Still Lesen \\
\hline $\begin{array}{c}\text { 2. Rezeptions- } \\
\text { durchgang }\end{array}$ & $\begin{array}{c}\text { Still Lesen und } \\
\text { Unterstreichen }\end{array}$ & $\begin{array}{c}\text { Still Lesen und } \\
\text { Reden über Text (UG) }\end{array}$ & $\begin{array}{c}\text { Still Lesen und } \\
\text { Schreiben }\end{array}$ \\
\hline
\end{tabular}

\begin{tabular}{|c|c|c|c|}
\hline & M 4 & M 5 & M 6 \\
\hline $\begin{array}{c}\text { 1. Rezeptions- } \\
\text { durchgang }\end{array}$ & Vorlesen & Vorlesen & Vorlesen \\
\hline $\begin{array}{c}\text { 2. Rezeptions- } \\
\text { durchgang }\end{array}$ & $\begin{array}{c}\text { Still Lesen und } \\
\text { Unterstreichen }\end{array}$ & $\begin{array}{c}\text { Still Lesen und } \\
\text { Reden über Text (UG) }\end{array}$ & $\begin{array}{c}\text { Still Lesen und } \\
\text { Schreiben }\end{array}$ \\
\hline
\end{tabular}




\begin{tabular}{|c|c|c|c|}
\hline & M 7 & M 8 & M 9 \\
\hline $\begin{array}{c}\text { 1. Rezeptions- } \\
\text { durchgang }\end{array}$ & $\begin{array}{c}\text { Aktivieren von } \\
\text { Vorwissen }\end{array}$ & $\begin{array}{c}\text { Aktivieren von } \\
\text { Vorwissen }\end{array}$ & Aktivieren von Vorwissen \\
\hline $\begin{array}{c}\text { 2. Rezeptions- } \\
\text { durchgang }\end{array}$ & $\begin{array}{c}\text { Still Lesen und } \\
\text { Unterstreichen }\end{array}$ & $\begin{array}{c}\text { Still Lesen und } \\
\text { Reden über Text (UG) }\end{array}$ & $\begin{array}{c}\text { Still Lesen und } \\
\text { Schreiben }\end{array}$ \\
\hline
\end{tabular}

Abb. 1: $\quad$ Modell der neun Methodenkombinationen bzw. Untersuchungseinheiten

UG: Unterrichtsgespräch

Anhand einer dieser Methodenkombinationen wurde pro Schulklasse ein Sachtext aus einem Schulbuch der Biologie rezipiert. Anschließend führten die Schülerinnen und Schüler einen Lesetest durch, der in Anlehnung an den Hamburger Lesetest HAMLET (Lehmann et al.: 1995) konzipiert wurde. ${ }^{4}$ Bei dem Test ging es um das Verstehen von Sachtexten, nicht um das Behalten. Daher durften die Schülerinnen und Schüler beim Ausfüllen des Lesetests im Untersuchungstext nachschauen.

\subsection{Erwartete Ergebnisse}

Die Studie sollte Aufschluss über die Leseleistung von Mädchen und Jungen in der 7. Klasse und über die geschlechtsspezifische Präferenz von Rezeptionsstrategien und deren Kombinationen erbringen. Aufgrund der Befunde der vorangegangenen Studie in der Grundschule 4. Klasse (Belgrad/Grütz/Pfaff 2003, Grütz 2004) wurden folgende Ergebnisse in der Sekundarstufe vermutet ${ }^{5}$ :

\section{1) Prognosen über Leseleistungen: Mädchen schneiden beim Rezipieren von Sachtexten} vermutlich geringfügig besser ab als Jungen. Dies dürfte in beiden Schultypen der Fall sein.

Im 4. Schuljahr wird in der Studie von Belgrad/Grütz/Pfaff (2003) eine Überlegenheit der Mädchen gegenüber den Jungen beim Verstehen von Sachtexten festgestellt. Mädchen bringen im Lesetest bei fast allen Methodenkombinationen signifikant bessere Leistungen (Grütz 2004: 35f.). Eine Untersuchung im 6. Schuljahr an Grundschulen in Brandenburg6 kommt zu ähnlichen Ergebnissen: Im 6. Schuljahr sind etwa $67 \%$ der Jungen und $77 \%$ der Mädchen in der Lage, altersentsprechende Texte sinnentnehmend zu lesen (ScheererNeumann 2003: 551). Auch in den PISA-Studien, in denen 15-jährige Schülerinnen und Schüler (in Deutschland 9. Klasse) getestet wurden, zeigen die Mädchen eine bessere Leseleistung bei kontinuierlichen Texten, wie z.B. Darlegungen (Stanat/Kunter 2001: 255). Ein ausgeglichenes Verhältnis zwischen den Geschlechtern stellt dagegen die IGLU-Studie für Viertklässler (2004) fest: Beim Leseverstehen von Informationstexten gibt es zugunsten der Mädchen geringfügige Unterschiede zwischen den Geschlechtern (Bos et al. 2003: 116).

\footnotetext{
${ }^{4}$ Der Untersuchungstext samt Anleitung für die Lehrkraft sowie der Lesetest sind zu finden im Forschungsbericht zur Studie: Grütz/Belgrad/Pfaff, 2004: 12ff. www.ph-weingarten.de/deutsch.

5 Diese Ergebnisse wurden in Grütz (2004), linguistik online 21/04: 23-46 vorgestellt.

${ }^{6}$ In Brandenburg ist die Grundschule sechsjährig.
} 
Aufgrund dieser Studien vermuteten wir ein besseres Abschneiden der Mädchen in der 7. Klasse.

Prognosen über erfolgreiches Leseverstehen sollten auch den Zusammenhang zwischen Interesse und Textverstehen berücksichtigen (Naceur 2001). Interessiert ein Thema, werden dazu auch freiwillig bzw. gerne Texte gelesen. Wissen wird aufgebaut, das vor dem Lesen aktiviert werden kann und beim Lesen Inferenzziehung ermöglicht. Daher sind bei Interesse bessere Leseergebnisse zu erwarten. Generell interessieren sich Mädchen und Jungen zwar für unterschiedliche Themen und Textsorten (Bertschi-Kaufmann 2002: 152f.). Das den Jungen nachgesagte Interesse für Sachthemen nimmt aber im Gegensatz zu früheren Untersuchungen ab (Bischoff/Heidtmann 2002b: 27ff.). Inwieweit Texte aus Schulbüchern auf das Interesse der Schülerinnen und Schüler treffen, ist fraglich. Bischoff und Heidtmann stellten zumindest für Schullektüre ein "mäßiges" Interesse bei Schülerinnen und Schülern fest (2002b: 27ff.). Im Zusammenhang mit der Studie zum Leseverstehen von Sachtexten in der Sekundarstufe von Grütz/Belgrad/Pfaff (2004) wurde eine Erhebung zum Interesse am Untersuchungstext ein Schulbuchtext zum Thema Herz - im Rahmen einer wissenschaftlichen Hausarbeit durchgeführt. Dabei wurde von Mädchen wie Jungen gleichermaßen Interesse am Thema des Textes geäußert. Nur ein Mädchen meinte, dass Biologie nicht ihr "Fall" sei (Demmer 2005). Insgesamt kann bei beiden Geschlechtern von einem gleichen Interesse am Thema ausgegangen werden, was den Verstehensprozess gleichermaßen positiv wie negativ beeinflusst. $^{7}$

Den Faktor Interesse berücksichtigend wurde vermutet, dass Mädchen, die im Allgemeinen besser als Jungen abschneiden, bei gleichem Interesse nur noch geringfügig bessere Leseleistungen erzielen als Jungen.

In der Sekundarstufe muss zudem das gegliederte Schulsystem Berücksichtigung finden: In der Hauptschule sind andere Leseleistungen zu erwarten als in der Realschule. Dies zeigt für die 15-Jährigen erneut die PISA-Studie 2003. Zwar sind in allen Schultypen alle Kompetenzstufen vertreten, doch gibt es signifikante Unterschiede im Durchschnittswert: Während in der Hauptschule mehr als die Hälfte aller Schülerinnen und Schüler lediglich die Kompetenzstufe 1 im Übergang zur Kompetenzstufe 2 erreichen, sind in der Realschule die Schülerinnen und Schüler mehrheitlich in der Lage, Aufgaben der Kompetenzstufe 3 zu lösen (PISA-Konsortium Deutschland (ed.) 2004: 105f.). Wir leiteten daraus für unsere Studie einen deutlichen Leistungsunterschied zwischen Hauptschule und Realschule ab.

2) Prognosen über Rezeptionsstrategien: Mädchen schneiden vermutlich bei mündlich orientierten Strategien am besten ab. Das sind Methodenkombinationen mit Aktivieren von Vorwissen im ersten und Reden über den Text im zweiten Rezeptionsdurchgang. Vermutlich

\footnotetext{
${ }^{7}$ Ein gleiches Interesse von Mädchen und Jungen am Untersuchungstext aus der Biologie zum Thema Herz kann auch aufgrund einer Untersuchung von Naceur (2001) angenommen werden. Er befragte Mädchen und Jungen nach ihrem Interesse an vier thematisch unterschiedlichen Texten. Bei einem Text aus der Biologie zum Gehirn bestand bei beiden Geschlechtern gleiches Interesse (2001: 119).
} 
kommen Mädchen auch mit dem Stilllesen zu guten Ergebnissen. Bei Jungen ist anzunehmen, dass sie ihre besten Ergebnisse erzielen, wenn sie den Text still durchlesen können.

Über geschlechtsspezifisch unterschiedliche Zugriffsweisen auf Sachtexte im Sinne einer besonderen Präferenz für Rezeptionsstrategien kam die Grundschul-Studie von Belgrad, Grütz/Pfaff (2003) zu folgenden Ergebnissen: In der 4. Klasse rezipieren die Mädchen Sachtexte besonders gut, wenn vor dem Lesen Vorwissen aktiviert oder wenn der Text still gelesen wird. Die Methodenkombinationen Aktivieren von Vorwissen und Reden führt bei ihnen zum besten Ergebnis. Bei dieser Methodenkombination gibt es auch den größten Unterschied in der Leseleistung zwischen den Geschlechtern zugunsten der Mädchen. Dies zeigt, dass Mädchen im späten Grundschulalter deutlich besser als die Jungen mit Verfahren umgehen können, bei denen es auf den verbalen Austausch ankommt (vgl. Tab. 7 im Anhang).

Bei den Jungen führen alle Methodenkombinationen mit dem Stilllesen als Verfahren des ersten Lesedurchgangs zu den besten Ergebnissen. Da mit dieser Methode auch die Mädchen gut abschneiden, ist zu schließen, dass dieses Verfahren für das Rezipieren von Sachtexten unabhängig vom Geschlecht eine genuine Methode ist. Das stille Durchlesen eines Textes dürfte leistungsstarken und -schwachen Leserinnen und Lesern entgegenkommen, da der Text nach der individuellen Organisation des Leseprozesses (Lesetempo, Vor- und Zurücklesen) rezipiert werden kann (Belgrad/Grütz/Pfaff 2003: 35) ${ }^{8}$. Jungen schneiden in der Grundschule auch dann überwiegend gut ab, wenn sie den Text unterstreichen können (vgl. Tab. 8 im Anhang).

Bei Vermutungen über den Erfolg bestimmter Rezeptionsstrategien ist der (individuelle) Lesesozialisationsprozess zu beachten: Kinder nähern sich in ihren Rezeptionsstrategien im Laufe ihrer Entwicklung nicht linear dem Lesen der Erwachsenen an, sondern bilden unterschiedliche Zugriffsweisen aus, die sie zeitweilig als ihre dominanten Strategien bevorzugen (Scheerer-Neumann 2003: 554). Scheerer-Neumann legt damit den Zusammenhang zwischen Alter bzw. Entwicklungsstand und bevorzugter Lesestrategie nahe. Lesesozialisation ist auch geschlechtsspezifisch determiniert. Dies äußert sich in einer unterschiedlichen Leseintensität und in einer unterschiedlichen Wahl von Themen und Textsorten (Bischoff/Heidtmann 2002, Eggert/Garbe 1995). Zu vermuten ist, dass aufgrund der geschlechtsspezifischen Sozialisation auch die Rezeptionsstrategien bei Mädchen und Jungen variieren.

\footnotetext{
8 In der die Sekundarstufenstudie ergänzenden Befragung von Schülerinnen und Schülern im Rahmen einer wissenschaftlichen Hausarbeit von Demmer (2005) wurde diese Annahme bestätigt.
} 


\section{Ergebnisse bei Mädchen und Jungen in der Sekundarstufe I - Hauptschulen und Realschulen 7. Klasse}

\subsection{Leseleistungen bei Mädchen und Jungen an Hauptschulen und Realschulen}

Ein wesentliches Ergebnis der Studie ist, dass es zwischen Mädchen und Jungen in der 7. Klasse innerhalb eines Schultyps keine Unterschiede in der Leseleistung gibt. Das trifft für die Hauptschulen und die Realschulen zu. Von fünf möglichen Punkten im Lesetest erreichen in den Hauptschulen die Mädchen im Durchschnitt 2,66 Punkte, die Jungen 2,71 Punkte. An den Realschulen erreichen die Mädchen 3,62 Punkte, die Jungen 3,60 Punkte. Mädchen und Jungen im Alter von etwa 13 Jahren $^{9}$ sind also bei gleichem Bildungsniveau gleichermaßen in der Lage, Sachtexten Informationen zu entnehmen. ${ }^{10}$

Dies ist gegenüber der Grundschul-Studie 4. Klasse von Belgrad/Grütz/Pfaff (2003) ein auffallendes Ergebnis, da dort die Mädchen signifikant besser abschnitten als die Jungen (Grütz 2004: 35ff.). Auch in den beiden PISA-Studien von 2000 und 2003, die in Bezug auf das Alter der Proband/innen einen sinnvollen Vergleich darstellen, bestätigt sich das Ergebnis unserer Studie nicht: Die Lesekompetenz der Mädchen ist signifikant höher als die der Jungen (PISA-Konsortium Deutschland 2004: 103). Das Ergebnis der vorliegenden SekundarstufenStudie kommt eher dem Befund der IGLU-Studie nahe, in der beim Lesen von Informationstexten die Mädchen nur unerheblich besser waren als die Jungen (vgl. Bos et al. 2003: 114).

Interpretation. Eine mögliche Erklärung für die Diskrepanz der vorliegenden Studie zur PISA-Studie durch die Berücksichtigung der bearbeiteten Textsorten ergibt keinen weiteren Aufschluss. In der PISA-Studie 2000 war die Geschlechterdifferenz nur bei diskontinuierlichen Textsorten zwischen den Geschlechtern ausgeglichen - z. T. waren hier die Jungen sogar etwas besser. Bei den kontinuierlichen Texten hingegen (z.B. Darlegungen, Erörterungen), worunter der Text der vorliegenden Studie einzuordnen ist, wurde den Mädchen eine weitaus höhere Lesekompetenz bestätigt als den Jungen (Stanat/Kunter 2001: 255).

Das gleichwertige Abschneiden der Schülerinnen und Schüler könnte auf die Faktoren Interesse und Motivation zurückgeführt werden. Das belegt die PISA-Studie 2000: Der Geschlechterunterschied zugunsten der Mädchen fällt nur noch sehr gering aus, wenn Mädchen und Jungen ähnliches Interesse am Lesen haben, er verschwindet ganz bei gleicher Freude am Lesen (Stanat/Kunter, 2001: 263; Grütz 2004: 26). Vermutlich waren Mädchen und Jungen gleichermaßen am Thema des Untersuchungstextes interessiert - unabhängig davon, wie hoch oder niedrig das Interesse war.

\footnotetext{
${ }^{9}$ In der 7. Klasse sind die Schüler/innen überwiegend 13 Jahre alt. Leichte Abweichungen von diesem Durchschnittsalter gibt es durch zu früh eingeschulte Schüler/innen oder durch solche, die eine Klasse wiederholen.

${ }^{10}$ Der Unterschied zwischen den beiden Bildungsniveaus Haupt- und Realschule ist hochsignifikant.
} 


\subsection{Rezeptionsstrategien von Mädchen an Hauptschulen und Realschulen ${ }^{11}$}

In diesem Kapitel werden die Ergebnisse von Mädchen in der Haupt- und Realschule verglichen, um herauszufinden, ob es geschlechtsspezifische Besonderheiten bei den Rezeptionsstrategien gibt, die vom Schultyp unabhängig sind.

\section{Ergebnisse bei den Methodenkombinationen}

Die Mädchen in der Hauptschule und in der Realschule haben eine Gemeinsamkeit: Beide erzielen mit der Methodenkombination M7 Aktivierung von Vorwissen und Unterstreichen eines ihrer signifikant besseren Ergebnisse. Das Besondere an diesem Ergebnis ist, dass die Mädchen in der Grundschule mit dieser Kombination am schlechtesten abschnitten.

\section{Ergebnisse bei den Verfahren des ersten Rezeptionsdurchgangs ${ }^{12}$}

Beim ersten Rezeptionsdurchgang gibt es bei den Mädchen zwischen beiden Schultypen deutliche Unterschiede. In der Hauptschule kommen die Mädchen mit dem Aktivieren von Vorwissen signifikant zu besten Ergebnissen. In der Realschule erzielen die Mädchen mit Stilllesen die signifikant besten Leseerfolge. Dieses Verfahren schneidet bei den Hauptschülerinnen hingegen am schlechtesten ab. Es ist zu vermuten, dass Mädchen in der Hauptschule eher aus bildungsfernen Elternhäusern kommen, in denen wenig gelesen wird. Daher tun sich Hauptschülerinnen schwer, Informationen selbstständig durch stilles Lesen Sachtexten zu entnehmen.

\section{Ergebnisse bei den Verfahren des zweiten Rezeptionsdurchgangs ${ }^{13}$}

Beim zweiten Rezeptionsdurchgang präferieren die Mädchen in der Hauptschule eindeutig das Reden über den Text. Schlecht schneiden sie ab, wenn sie aus dem Text Wörter herausschreiben müssen. ${ }^{14}$ Bei den Realschülerinnen ist es hingegen gleichgültig, wie sie den Sachtext im zweiten Lesedurchgang rezipieren. Sie erzielen gleiche Ergebnisse, unabhängig davon, ob sie Wörter unterstreichen, herausschreiben oder über den Text reden.

Zusammenfassend ist im Vergleich zwischen Hauptschülerinnen und Realschülerinnen festzustellen: Hauptschülerinnen sind in der 7. Klasse nicht in der Lage, selbstständig einem Sachtext Informationen zu entnehmen. In beiden Rezeptionsdurchgängen schneiden bei ihnen Verfahren am besten $a b$, in denen ein Gespräch geführt wird: wenn vor der Textbegegnung im Unterrichtsgespräch Vorwissen aktiviert wird und wenn im Anschluss an die Textlektüre über den Text geredet wird. Diese Methodenkombination ist laut Leseforschung zwar ein

\footnotetext{
11 Siehe auch Tab. 2 im Anhang.

12 Es wurden alle Methodenkombinationen mit dem gleichen ersten Rezeptionsdurchgang gruppiert, also alle Kombinationen mit Stillesen (M1, M2, M3), Vorlesen (M4, M5, M6) und Aktivieren von Vorwissen (M7, M8, M9).

13 Es wurden alle Methodenkombinationen mit dem gleichen zweiten Rezeptionsdurchgang gruppiert, also alle Kombinationen mit Unterstreichen (M1, M4, M7), Reden (M2, M5, M8) und Schreiben (M3, M6, M9).

${ }^{14}$ Zwischen dem Reden und dem Schreiben ist der Unterschied signifikant.
} 
strategischer Zugriff auf Texte, den geübte Leser/innen anwenden. Zu beachten sind aber zwei Befunde: Hauptschülerinnen haben insgesamt schlechte Leseleistungen und sie schneiden beim Stilllesen signifikant schlechter ab als beim Aktivieren von Vorwissen. Dies lässt vermuten, dass das gute Ergebnis beim Aktivieren von Vorwissen eher ein Ausdruck mangelnder Lesefähigkeit ist und die Hauptschülerinnen durch das Unterrichtsgespräch die benötigte Unterstützung durch die Lehrkraft bekommen.

Realschülerinnen sind in der 7. Klasse besser als Hauptschülerinnen befähigt, selbstständig mit einem Sachtext umzugehen und Informationen zu entnehmen. Sie erzielen die besten Ergebnisse, wenn sie im ersten Rezeptionsdurchgang den Text still durchlesen. Beim zweiten Rezeptionsdurchgang kommen sie mit allen Verfahren zu gleich guten Ergebnissen. Daraus lässt sich schließen, dass für Mädchen mit einer höheren Lesekompetenz der erste Rezeptionsdurchgang entscheidend für das Textverstehen ist.

Schultyp übergreifend konnten also nur zwei Gemeinsamkeiten bei den Mädchen festgestellt werden. Erstens erzielen mit der Kombination der beiden Verfahren Aktivieren von Vorwissen und Unterstreichen Mädchen in beiden Schultypen gute Ergebnisse. Zweitens führt das Reden über den Text als elaborative Textverarbeitungsstrategie bei beiden zu guten Verstehensleistungen. Dabei ist dieses Verfahren bei den Hauptschülerinnen das signifikant beste, bei den Realschülerinnen hingegen ist es gleichrangig mit den anderen getesteten Verfahren.

\subsection{Rezeptionsstrategien von Jungen an Hauptschulen und Realschulen ${ }^{15}$}

In diesem Kapitel werden die Ergebnisse von Jungen in der Haupt- und Realschule verglichen, um herauszufinden, ob es geschlechtsspezifische Besonderheiten bei den Rezeptionsstrategien gibt, die vom Schultyp unabhängig sind.

\section{Ergebnisse bei den Methodenkombinationen}

Bei den Methodenkombinationen konnte bei den Haupt- und Realschülern keine Gemeinsamkeit festgestellt werden.

\section{Ergebnisse bei den Verfahren des ersten Rezeptionsdurchgangs}

Beim ersten Rezeptionsdurchgang ist bei den Jungen an der Haupt- und Realschule ein kleinster gemeinsamer Nenner festzustellen: Das stille Durchlesen eines Sachtextes führt bei beiden zu guten Leseergebnissen. Bei beiden Gruppen gibt es jedoch - auch das eine Gemeinsamkeit - keine signifikanten Unterschiede zwischen den Verfahren des ersten Rezeptionsdurchgangs. Das Stilllesen ist also tendenziell eine für Jungen beider Schultypen geeignete Methode.

\footnotetext{
${ }^{15}$ Siehe auch Tab. 3 im Anhang
} 


\section{Ergebnisse bei den Verfahren des zweiten Rezeptionsdurchgangs}

Beim zweiten Rezeptionsdurchgang gibt es mehr Gemeinsamkeiten zwischen Jungen an Haupt- und Realschulen. Das Reden über den Text erzielt bei beiden die besten Verstehensleistungen. ${ }^{16}$ Als das signifikant schlechteste Verfahren stellt sich bei allen Jungen das Schreiben heraus. Dieses Ergebnis lässt vermuten, dass Jungen unabhängig von der Bildungsschicht in diesem Alter ungern schreiben und dass sie dadurch beim Leseverstehen behindert werden.

Zusammenfassend lässt sich bei den Jungen beider Schultypen eine gewisse Präferenz für das Stillesen im ersten Rezeptionsdurchgang feststellen. Im zweiten Rezeptionsdurchgang erzielen die Jungen mit dem Reden über den Text die besten Ergebnisse. Dies gilt insbesondere für die Jungen an der Hauptschule. Das signifikant schlechteste Leseergebnis haben alle Jungen, wenn sie aus dem Text Wörter herausschreiben sollen.

\subsection{Zusammenfassung: Rezeptionsstrategien bei Mädchen und Jungen an Hauptschulen und Realschulen}

In der Sekundarstufe 7. Klasse lassen sich folgende Besonderheiten beim Leseverstehensprozess zur Informationsentnahme aus Sachtexten feststellen:

Auffallend sind folgende Ergebnisse des ersten Rezeptionsdurchgangs: Das stille Durchlesen eines Textes erzielt bei vielen Schülerinnen und Schülern gute bis sehr gute Verstehensleistungen.

- $\quad$ Realschülerinnen schneiden mit Stilllesen signifikant am besten ab.

- $\quad$ Bei allen Jungen an Haupt- und Realschulen ist das stille Lesen ein Verfahren, das auch zu einem guten Leseerfolg führt. ${ }^{17}$

- $\quad$ Nur die Mädchen an der Hauptschule schneiden mit diesem Verfahren schlecht ab.

Daraus ist zunächst zu schließen, dass das Verfahren, einen Sachtext still durchzulesen, für diese Textsorte geschlechtsunabhängig eine geeignete Strategie zu sein scheint. Die Anwendbarkeit dieses Verfahrens will aber offensichtlich gelernt sein. Darauf verweisen die unterschiedlichen Ergebnisse zwischen den Haupt- und Realschülerinnen beim stillen Lesen.

\footnotetext{
16 Bei den Jungen an der Hauptschule ist dies das signifikant beste Verfahren. Bei den Realschülern führt Reden über den Text nicht ausschließlich zum Verstehenserfolg, sondern es ist gleichwertig mit dem Unterstreichen von Textstellen.

17 Bei den Jungen an der Hauptschule führt neben dem Stilllesen auch das Vorlesen des Textes durch die Lehrkraft zu guten Ergebnissen, bei den Jungen an der Realschule das Aktivieren von Vorwissen.
} 
Auffallend sind folgende Ergebnisse des zweiten Rezeptionsdurchgangs:

- $\quad$ Schreiben schneidet bei allen Jungen am schlechtesten ab. ${ }^{18}$

- $\quad$ Unter den Mädchen schneidet Schreiben nur in der Hauptschule schlecht ab.

- $\quad$ Bei den Mädchen in der Realschule ist dieses Verfahren den anderen gleichgestellt.

Aus den Ergebnissen zum Stilllesen und zum Schreiben kann man folgern: Die Rezeptionsstrategien sind nur bedingt abhängig vom Geschlecht. Es kommt ein zweiter Faktor hinzu, der des Bildungsniveaus. Dies lässt sich an der Kulturtechnik Schreiben nachvollziehen. Wörter herauszuschreiben ist ein als aufwändig empfundenes Verfahren. Dass insbesondere Mädchen mit einem höheren Bildungsniveau eher damit umgehen können, kann damit zu tun haben, dass Mädchen die Schulnormen besser erfüllen als Jungen (Kotthoff 2003; Rüegg 1995). ${ }^{19}$ Wie gut sie das tun, hängt schließlich vom Bildungsgrad und vermutlich auch vom Elternhaus $a b^{20}$. Bildungsnahe Elternhäuser pflegen die Kultur des Schriftlichen - Lesen und Schreiben - besser als bildungsferne Elternhäuser. Die Schule war bisher offenbar nicht in der Lage, das sprachliche Defizit bildungsferner Elternhäuser bei den Kindern auszugleichen. In der Hauptschule müsste verstärkt das Einüben schriftorientierter Textverarbeitungsstrategien fokussiert werden.

In diesem Kapitel wurde die Gesamtpopulation der Studie - alle Mädchen gegenüber allen Jungen - betrachtet. Eindeutige Aussagen über präferierte Rezeptionsstrategien sind aber nur zu treffen, wenn ein Schultyp differenziert betrachtet wird. In den beiden folgenden Kapiteln geht es um die Ergebnisse von Mädchen und Jungen im jeweiligen Schultyp. Aus diesen Ergebnissen können dann auch Schlüsse für einen geschlechtsspezifisch differenzierenden Unterricht gezogen werden.

\footnotetext{
18 In der Hauptschule besteht zwischen Reden (bestes Ergebnis) und Schreiben (schlechtestes Ergebnis) ein hochsignifikanter Unterschied; in der Realschule ist der Unterschied nur signifikant. In der Hauptschule besteht auch ein signifikanter Unterschied zum Unterstreichen (zweitbeste Rezeptionsstrategie), in der Realschule ist dieser Unterschied nur annähernd signifikant (Vgl. Tab. 3 im Anhang).

19 Dass Mädchen die Schulnormen besser erfüllen als Jungen, darauf verweist auch der prozentuale Anteil der Geschlechter in den Schultypen: Mädchen sind an den Realschulen überproportional vertreten, Jungen an den Hauptschulen. Vgl. hierzu auch Tab. 1 im Anhang.

20 Die PISA-Studie 2003 hat erneut den engen Zusammenhang zwischen sozialer Herkunft und Bildungsniveau festgestellt (PISA-Konsortium Deutschland 2004:243ff).
} 


\section{Rezeptionsstrategien von Mädchen und Jungen in der Hauptschule}

\subsection{Die wichtigsten Ergebnisse im Überblick}

Beim Vergleich der Ergebnisse von Mädchen und Jungen in der Hauptschule sind vier wesentliche Ergebnisse festzuhalten:

1. Bei den Leseleistungen gibt es in dieser Untersuchung insgesamt keinen Unterschied zwischen Mädchen und Jungen. Sie schneiden in der erreichten Punktezahl etwa gleich $\mathrm{ab}$.

2. Im ersten Lesedurchgang favorisieren Mädchen und Jungen jedoch unterschiedliche Methoden: Jungen schneiden mit den Verfahren Stillesen und Vorlesen signifikant besser ab als Mädchen. Mädchen schneiden mit dem Aktivieren von Vorwissen tendenziell besser ab als Jungen.

3. Im zweiten Rezeptionsdurchgang haben Mädchen und Jungen gleiche Präferenzen für bestimmte Methoden. Bei beiden führt das Reden über den Text zu den besten, das Herausschreiben von Wörtern zu den schlechtesten Leseleistungen.

4. Bei beiden Geschlechtern führt die Methodenkombination M8 Aktivierung von Vorwissen und Reden zu einem der besten Ergebnisse. Diese Kombination scheint also in der Hauptschule geschlechtsunabhängig bei der Rezeption von Sachtexten besonders geeignet zu sein.

\subsection{Ergebnisse von Mädchen und Jungen im Vergleich}

Bei den Ergebnissen von Mädchen und Jungen in der Hauptschule ergeben sich vier grundlegende Befunde:

\section{Befund: Mädchen und Jungen erbringen gleiche Leseleistungen.}

In dieser Studie konnten beim Verstehen von Sachtexten zwischen den Geschlechtern keine Unterschiede festgestellt werden. Die Mädchen erzielen bei fünf zu erreichenden Punkten im Durchschnitt 2,66 Punkte, die Jungen 2,71. Der Unterschied ist nicht signifikant. Lediglich bei einer Methodenkombination, M5 Vorlesen und Reden, schneiden die Jungen signifikant besser ab als die Mädchen (vgl. Tab. 5 im Anhang). 


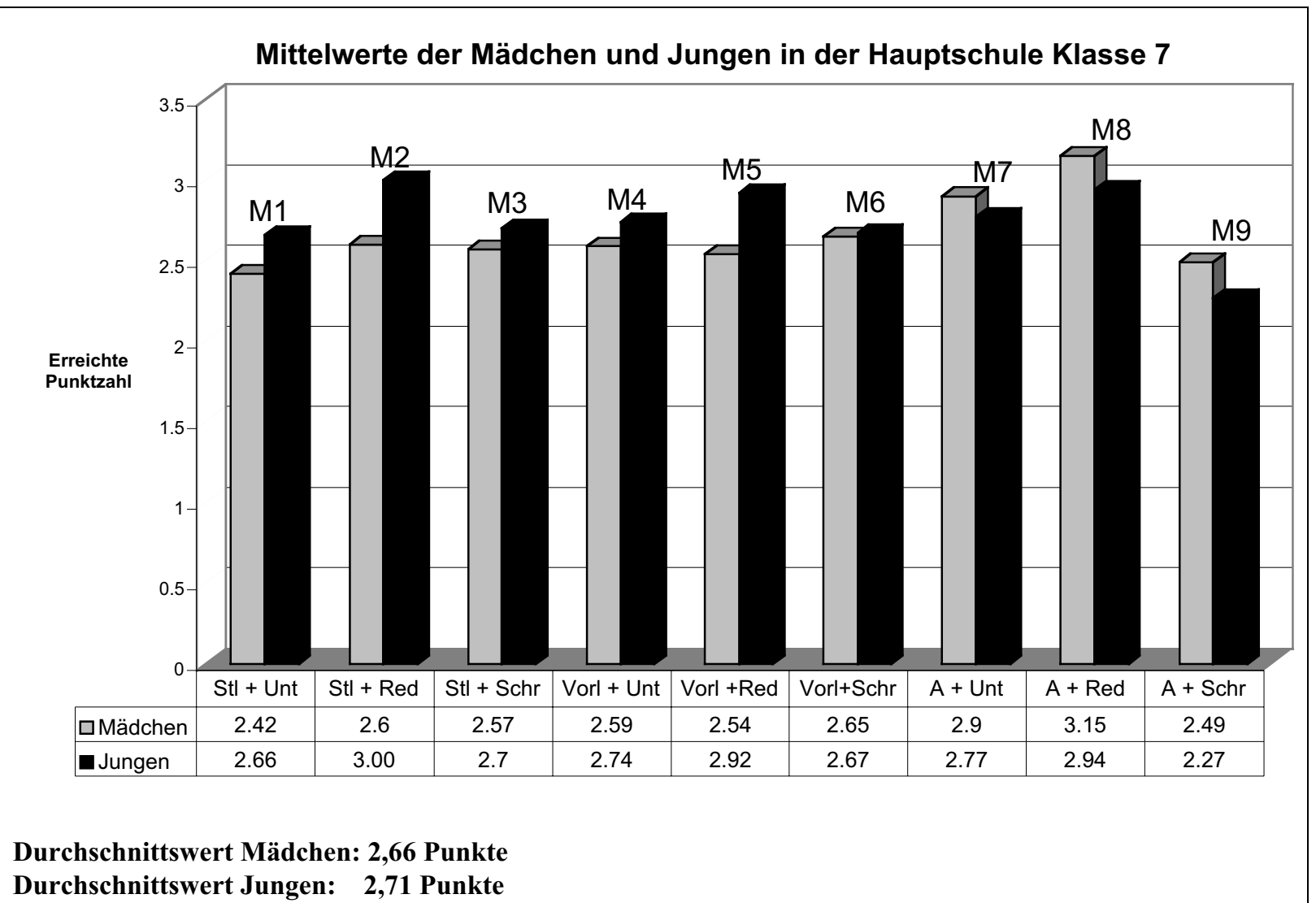

Abb. 2: Vergleich der Mittelwerte bei Mädchen und Jungen in der Hauptschule

\section{Befund: Mädchen und Jungen präferieren in der Hauptschule unterschiedliche}

Rezeptionsstrategien bei der ersten Textbegegnung.

Im ersten Rezeptionsdurchgang präferieren Mädchen und Jungen unterschiedliche Rezeptionsstrategien. Jungen schneiden bei Kombinationen mit Stilllesen und mit Vorlesen signifikant besser ab als Mädchen. Mädchen schneiden immer dann besser als Jungen ab, wenn vor dem Lesen das Vorwissen aktiviert wird. Dieser Unterschied ist fast signifikant (p: 0,07) (Abb. 3).

Beim Vergleich der einzelnen Methodenkombinationen (Abb. 2) zeigt sich folgender Unterschied: Bei allen Kombinationen mit dem Stilllesen weisen die Jungen bessere Ergebnisse auf (M1 - M3); ebenso bei zwei Kombinationen mit dem Vorlesen: M4 Vorlesen und Unterstreichen und M5 Vorlesen und Reden. Bei M5 Vorlesen und Reden liegt sogar ein signifikanter Unterschied zugunsten der Jungen vor. Die einzige Methodenkombination, bei der beide Geschlechter gleiche Ergebnisse erzielen, ist M6 Vorlesen und Schreiben. Umgekehrt weisen die Mädchen bei allen Methodenkombinationen mit dem Aktivieren von Vorwissen (M7 - M9) bessere Ergebnisse auf als die Jungen. 


\begin{tabular}{|c|c|c|c|c|}
\hline $\begin{array}{l}\text { Verfahren des ersten } \\
\text { Rezeptionsdurchgangs }\end{array}$ & Stilllesen $^{21}$ & Vorlesen $^{22}$ & $\begin{array}{c}\text { Aktivieren von } \\
\text { Vorwissen }^{23}\end{array}$ & Gesamt \\
\hline Mädchen, Hauptschule & 2,51 & 2,60 & 2,81 & 2,66 \\
\hline Jungen, Hauptschule & 2,74 & 2,79 & 2,63 & 2,71 \\
\hline $\begin{array}{c}\text { p-Wert } \\
\text { Unterschiede zwischen } \\
\text { Mädchen - Jungen }\end{array}$ & $\begin{array}{c}\text { Stilllesen } \\
0,04^{*} \\
\text { (Jungen besser) }\end{array}$ & $\begin{array}{c}\text { Vorlesen } \\
0,05^{*} \\
\text { (Jungen besser) }\end{array}$ & $\begin{array}{c}\text { Aktivieren von } \\
\text { Vorwissen } \\
0,07 \text { (fast } * \text { ) } \\
\text { (Mädchen } \\
\text { besser) }\end{array}$ & $\begin{array}{l}\text { Jungen } \\
\text { signifikant } \\
\text { besser: Stl, V } \\
\text { Mädchen } \\
\text { besser: Akt. } \\
\text { Vorw. }\end{array}$ \\
\hline
\end{tabular}

Abb. 3: $\quad$ Vergleich der Methodenkombinationen mit Stillesen, Vorlesen, Aktivieren von Vorwissen zwischen Mädchen und Jungen an Hauptschulen

*: signifikant, wenn $\mathrm{p} \leq 0,05$. n. s.: nicht signifikant

Interpretation. Der Befund zu den unterschiedlichen Präferenzen bei den Verfahren des ersten Rezeptionsdurchgangs weist darauf hin, dass Mädchen und Jungen z.T. über unterschiedliche Verhaltensgewohnheiten und Fähigkeiten verfügen, die sich beim Leseverstehen auswirken. Ein Vergleich mit den Befunden der vorangegangenen GrundschulStudie 4. Klasse (Belgrad/Grütz/Pfaff 2003) zeigt, inwieweit diese Ausprägungen schon im Alter von etwa zehn Jahren vorliegen (Grütz 2004: 36f.).

Bei den Jungen wurde bereits in der Grundschul-Studie 4. Klasse die Präferenz für Methodenkombinationen mit dem Stilllesen deutlich. ${ }^{24}$ In der Studie zur Sekundarstufe hat sich diese Präferenz der Jungen dahingehend verstärkt, dass sie in der Hauptschule sogar bessere Leseleistungen erbringen als die Mädchen. Kombinationen mit dem Vorlesen führten bei den Jungen in der Grundschule zu divergenten Ergebnissen. ${ }^{25}$ Bei Hauptschülern ist die Bedeutung des Vorlesens gewachsen. Die Geschlechterunterschiede sind aufgehoben bzw. haben sich sogar zugunsten der Jungen verändert.

Dass Jungen gegenüber den Mädchen beim Stilllesen und beim Vorlesen besser abschneiden, kann kognititionspsychologisch erklärt werden: Das stille Lesen und das Vorlesen sind

\footnotetext{
${ }^{21}$ Stilllesen $=$ Methodenkombinationen 1, 2, 3

22 Vorlesen = Methodenkombinationen 4, 5, 6

23 Aktivieren von Vorwissen = Methodenkombinationen 7, 8, 9

24 Methodenkombinationen mit Stilllesen schnitten auch bei den Mädchen gut ab.

25 Die Kombination Vorlesen und Unterstreichen war gleichwertig mit den guten Ergebnissen beim stillen Lesen. Die Methodenkombination M6 Vorlesen und Schreiben, die in der vorliegenden Studie als einzige Methodenkombination zu gleichen Ergebnissen bei beiden Geschlechtern führt, wies in der Grundschul-Studie 4. Klasse einen hochsignifikanten Unterschied zwischen Mädchen und Jungen auf. Die Jungen erzielten hierbei ihr schlechtestes Ergebnis.
} 
Verfahren, die eher den Bottom-up-Prozess beim Aufbau eines mentalen Modells ansprechen. Die Information aus dem Text wird beim Lesen oder beim Zuhören sukzessive rezipiert. Es baut sich allmählich eine propositionale Textrepräsentation in der Kognition des Lesers auf. Einigkeit besteht in der Kognitionspsychologie und in der Leseforschung darüber, dass das Textverstehen als interaktive Konstruktion von Bedeutung verläuft. Doch ist unklar, welche Prozesse vorrangig wirken. Nach Perfettis Theorie der verbalen Effizienz (1985) ist überwiegend der Bottom-up-Prozess an der Bedeutungskonstruktion beteiligt. Ein sicheres Worterkennen führt zu guten Leseleistungen (vgl. Belgrad/Grütz/Pfaff 2003: 7) Diese Zugriffsweise auf Sachtexte scheint bei Jungen eher ausgeprägt zu sein (Grütz 2004: 37). ${ }^{26}$

Bei den Mädchen ist bereits in der Grundschul-Studie zu erkennen, dass sie mit dem Aktivieren von Vorwissen tendenziell besser umgehen können als die Jungen (Grütz 2004:36; 40). ${ }^{27}$ In der vorliegenden Studie zur Sekundarstufe hat sich die vorhandene Präferenz der Mädchen für Methodenkombinationen mit dem Aktivieren von Vorwissen bestätigt und sogar verstärkt. Sie schneiden damit bei allen kombinierten Verfahren (Unterstreichen, Reden, Schreiben) besser als die Jungen ab (Abb. 2). Hiermit scheint sich die in der PISA-Studie getroffene Feststellung zu bestätigen, dass Mädchen eher in der Lage sind als Jungen, "Verknüpfungen mit den Inhalten und dem eigenen Vorwissen herzustellen" (Stanat/Kunter 2001: 266)..$^{28}$

\section{Befund: $\quad$ Mädchen und Jungen präferieren in der Hauptschule gleiche Rezeptions- strategien bei der vertieften Auseinandersetzung mit dem Text.}

Im zweiten Rezeptionsdurchgang ziehen Mädchen wie Jungen das Reden über den Text vor. Kombinationen mit dem Reden schneiden bei beiden Geschlechtern am besten ab (Abb. 4). Auch das Verfahren mit den schlechtesten Ergebnissen ist bei beiden Geschlechtern gleich: Kombinationen mit dem Schreiben schneiden bei Mädchen und Jungen gleichermaßen am schlechtesten ab.

Interpretation. Der Befund, dass im zweiten Rezeptionsdurchgang beide Geschlechter gleichermaßen das Reden über den Text bevorzugen, wirft bei der Interpretation Probleme auf: Mehrere Studien belegen, dass Jungen im Unterricht eher unaufmerksam und unruhig sind (z.B. Rüegg 1995, van Alphen 1996, Fuchs 2001). Das Unterrichtsgespräch bietet Raum für dieses Verhalten. Es wird angenommen, dass Jungen unter Rahmenbedingungen, die es

\footnotetext{
${ }^{26}$ Es ist allerdings zu betonen, dass die Ergebnisse bezüglich des Stilllesens und des Vorlesens nur im Vergleich zu den Mädchen und im Vergleich zu den Ergebnissen der Grundschul-Studie besondere Beachtung finden. Vergleicht man diese beiden Verfahren innerhalb der Gruppe der Jungen mit dem Ergebnis des Aktivierens von Vorwissen, ergibt sich nur ein tendenzieller Unterschied, der nicht signifikant ist. Vgl. Kap. 3.4, Abb.10.

27 Einen signifikanten Unterschied gab es in der Grundschul-Studie bei M8 Aktivierung von Vorwissen und Reden. Etwas besser waren die Mädchen bei M7 Aktivierung von Vorwissen und Unterstreichen. Bei M9 Aktivierung von Vorwissen und Schreiben hatten allerdings die Jungen (als einziger Methodenkombination) etwas bessere Ergebnisse.

28 Trotz dieser kognititionspsychologischen Begründung steht die obige Überlegung im Raum, ob das das Vorwissen aktivierende Unterrichtsgespräch von den Hauptschülerinnen nicht eher als "Ersatz" für bzw. Komplement zu selbstständigem Lesen genutzt wurde
} 
ihnen ermöglichen, in Stillarbeit konzentriert zu arbeiten, besser lernen (Grütz 2004: 38). Diese Vermutung wird durch das gute Abschneiden des stillen Lesens im ersten Rezeptionsdurchgang bestätigt. Das eindeutig gute Abschneiden des Redens über den Text spricht aber gegen diese Vermutung.

\begin{tabular}{|c|c|c|c|c|}
\hline $\begin{array}{l}\text { Verfahren des zweiten } \\
\text { Rezeptionsdurchgangs }\end{array}$ & $\operatorname{Reden}^{29}$ & Unterstreichen $^{30}$ & Schreiben $^{31}$ & Gesamt \\
\hline $\begin{array}{l}\text { Mittelwerte } \\
\text { Mädchen, Hauptschule }\end{array}$ & 2,81 (1.) & 2,65 (2.) & 2,57 (3.) & 2,66 \\
\hline $\begin{array}{l}\text { Mittelwerte } \\
\text { Jungen, Hauptschule }\end{array}$ & 2,94 (1.) & 2,73 (2.) & $2,51(3)$. & 2,71 \\
\hline $\begin{array}{c}\text { p-Wert } \\
\text { Unterschiede zwischen } \\
\text { Mädchen - Jungen }\end{array}$ & $\begin{array}{c}\text { Reden } \\
0,24\end{array}$ & $\begin{array}{c}\text { Unterstreichen } \\
0,42\end{array}$ & $\begin{array}{c}\text { Schreiben } \\
0,58\end{array}$ & $\begin{array}{c}\text { keine } \\
\text { signifikanten } \\
\text { Unterschiede } \\
\text { zwischen den } \\
\text { Geschlechtern }\end{array}$ \\
\hline
\end{tabular}

\section{Abb. 4: $\quad$ Vergleich der Methodenkombinationen mit Reden, Unterstreichen, Schreiben nach Geschlecht (Hauptschule)}

n. s.: nicht signifikant *: signifikant, wenn $\mathrm{p} \leq 0,05$.

Es ist zu fragen, inwiefern die Jungen (und auch die Mädchen) von dem Verfahren Reden über den Text profitieren. Zu bedenken sind dabei die generell schwachen Leseleistungen. Vermutlich erhalten Schülerinnen und Schüler beim Reden über den Text durch die Lehrkraft Unterstützung bei der Textrezeption. Das Ziel eines Unterrichtsgesprächs ist es im Allgemeinen, auf richtige Lösungen hinzuführen. Wie zielorientiert dies geschieht, liegt letztlich an der Lehrkraft. ${ }^{32}$ Beim Unterstreichen und Schreiben müssen die Schülerinnen und Schüler selbstständig agieren. Die Leseverstehensleistungen sind bei diesen Verfahren schwächer.

\section{Befund: Bei beiden Geschlechtern führt die Methodenkombination M8 Aktivierung von Vorwissen und Reden zu einem der besten Ergebnisse.}

Diese Kombination scheint in der Hauptschule geschlechtsunabhängig bei der Rezeption von Sachtexten besonders geeignet zu sein. Der Grund mag sein, dass beide Verfahren durch das Gespräch verknüpft sind und sich ergänzend aufeinander beziehen. Die Schülerinnen und Schüler erhalten dadurch beim Leseverstehen konsistente Unterstützung. Offensichtlich ist es bei Schülerinnen und Schülern mit einer eher geringen Lesekompetenz wichtig, den Rezeptionsprozess durch mündliche, elaborative Verfahren extern zu unterstützen.

\footnotetext{
${ }^{29}$ Reden $=$ Methodenkombinationen 2, 5, 8

${ }^{30}$ Unterstreichen $=$ Methodenkombinationen 1, 4, 7

31 Schreiben = Methodenkombinationen 3, 6, 9

32 Im Test lautete die Anweisung an die Lehrkraft: Lehrkraft sagt: Was war für euch am wichtigsten / am interessantesten? Woran erinnert ihr euch? Wenn ihr wollt, könnt ihr im Text nachschauen. Es ist aber nicht unbedingt nötig.
} 
Zusammenfassung: In diesem Kapitel wurden die Unterschiede zwischen Mädchen und Jungen im Leseverstehen behandelt. Es ging darum, welches Geschlecht welche Methodenkombinationen bzw. welches Einzelverfahren vorzieht. Ein wichtiges Ergebnis beim ersten Rezeptionsdurchgang ist: Wenn ein Sachtext in der Schule still gelesen werden soll oder wenn die Lehrkraft ihn vorliest, schneiden die Jungen besser ab als die Mädchen. Soll aber vor der ersten Textbegegnung das Vorwissen aktiviert werden, schneiden die Mädchen besser ab als die Jungen. Bei den Jungen scheint im ersten Rezeptionsdurchgang also eher der Bottom-up-Prozess zu wirken, bei Mädchen der Top-down-Prozess. Ein wichtiges Ergebnis beim zweiten Rezeptionsdurchgang ist: Sowohl bei Mädchen als auch bei Jungen führt die elaborative Strategie Reden über den Text zu den besten Ergebnissen, wohingegen die reduktiv-organisatorischen Strategien Unterstreichen und Herausschreiben von Wörtern signifikant schlechter abschneiden. Dies zeigt, dass schriftorientierte Verfahren der Textverarbeitung bei beiden Geschlechtern an der Hauptschule große Schwierigkeiten beim Textverstehen hervorrufen.

In den beiden folgenden Kapiteln werden die Ergebnisse nach Geschlechtern getrennt behandelt und zu interpretieren versucht.

\subsection{Ergebnisse bei den Mädchen in der Hauptschule}

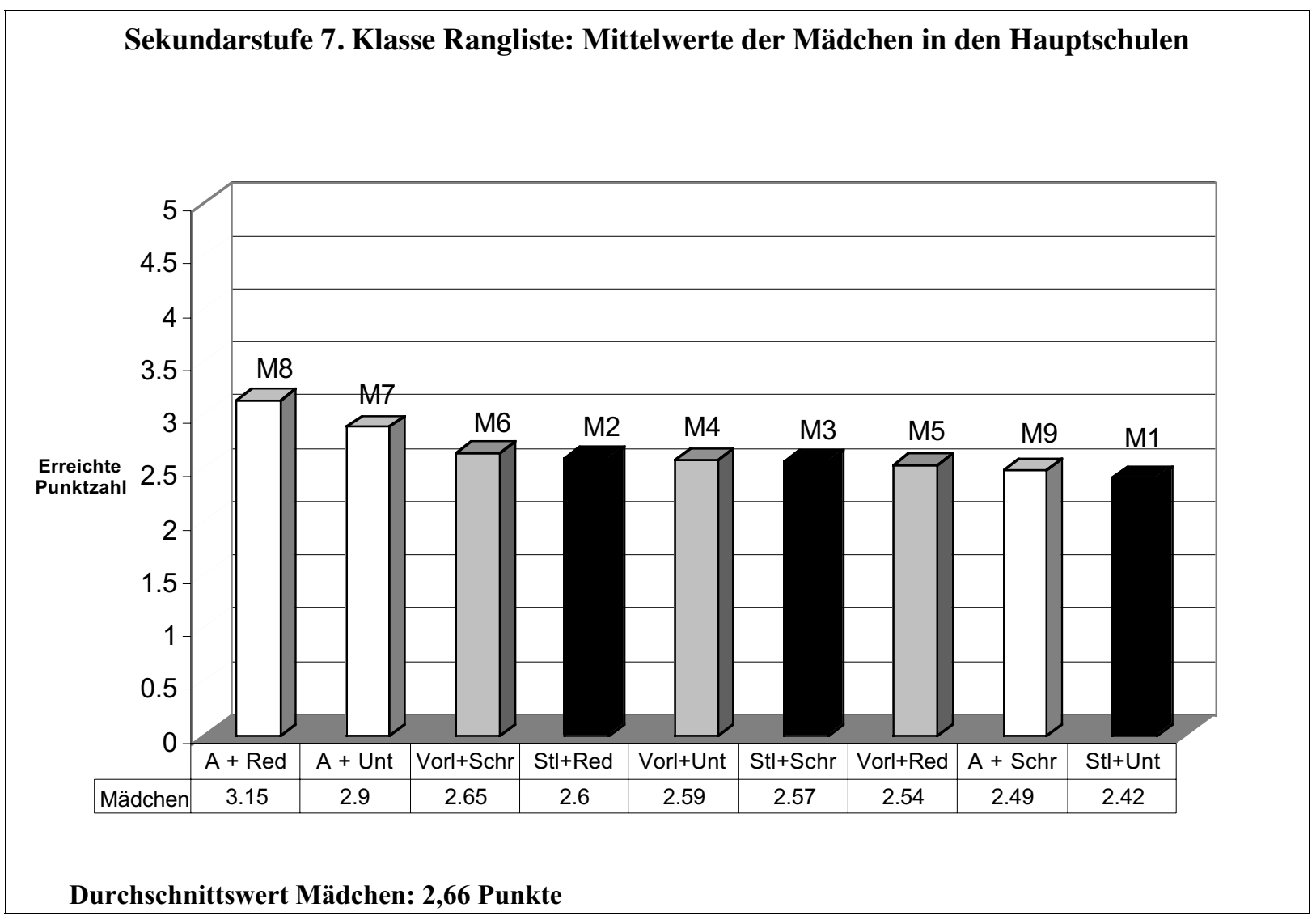

Abb. 5: $\quad$ Rangfolge der Methodenkombinationen bei Mädchen in der Hauptschule 7. Klasse 


\section{Ergebnisse bei den Methodenkombinationen (Abb. 5)}

Die beiden besten Ergebnisse erzielen die Mädchen mit den Methodenkombinationen M8 Aktivierung von Vorwissen und Reden und M7 Aktivierung von Vorwissen und Unterstreichen. Bei diesen Methodenkombinationen sind deutlich bessere Ergebnisse als bei den anderen Methodenkombinationen erzielt worden. Alle anderen Methodenkombinationen unterscheiden sich im Ergebnis kaum. ${ }^{33}$ Sie scheinen austauschbar zu sein.

Überraschend ist das Abschneiden der Methodenkombination M9 Aktivierung von Vorwissen und Schreiben (Rang 8). Es wäre zu erwarten gewesen, dass alle Verfahren, die mit dem Aktivieren von Vorwissen kombiniert werden, gute Ergebnisse erzielen. Die Kombination M9 Aktivierung von Vorwissen und Schreiben ist jedoch signifikant schlechter als M8 und M7. Vermutlicht hemmt das Schreiben in der Hauptschule den Verstehenserfolg.

Eine weitere Überraschung ist, dass die Methodenkombination M1 Stilllesen und Unterstreichen, die in der Grundschul-Studie 4. Klasse (Belgrad/Grütz/Pfaff 2003) bei den Mädchen mit zu den erfolgreichsten Methodenkombinationen gehört hatte, in der Sekundarstufe ein unterdurchschnittliches Ergebnis aufweist.

\section{Ergebnisse bei den Verfahren des ersten Rezeptionsdurchgangs (Abb. 6)}

Im ersten Rezeptionsdurchgang erzielen die Mädchen mit Aktivieren von Vorwissen die signifikant besseren Leseleistungen. Dieses Verfahren schneidet gegenüber dem Stilllesen und dem Vorlesen signifikant besser ab. ${ }^{34}$

\begin{tabular}{|c|c|c|c|c|}
\hline $\begin{array}{c}\text { Mittelwerte } \\
\text { Mädchen, } \\
\text { Hauptschule }\end{array}$ & $\begin{array}{c}\text { Aktivieren von } \\
\text { Vorwissen } \\
2,81(1 .)\end{array}$ & $2,60(2)$. & 2,51 (3.) & Stillesen \\
\hline p-Werte & $\begin{array}{l}\text { Aktivieren von Vorwissen }>\text { Vorlesen } 0,04 * \\
\text { Aktivieren } \text { von Vorwissen }>\text { Stillesen } 0,01 * \\
\text { Verfahren im } \\
\text { Vergleich }\end{array}$ & $\begin{array}{l} \\
\text { Vorlesen }>\text { Stillesen } 0,40 \mathrm{n} \text {. s. }\end{array}$ & \\
\hline
\end{tabular}

\section{Abb. 6: Verfahren des ersten Rezeptionsdurchgangs bei Mädchen an der Hauptschule}

n. s.: nicht signifikant $*$ : signifikant, wenn $\mathrm{p} \leq 0,05$.

33 Zwischen den Rängen 3-9 gibt es keine signifikanten Unterschiede. Ausführlich hierzu vgl. Grütz/Belgrad/Pfaff (2004): Forschungsbericht Teil B. Kap. 2.3. In: http://www.ph-weingarten.de > Lehre und Forschung $>$ Fakultät II $>$ Deutsch.

34 Das gute Ergebnis des Aktivierens von Vorwissen wird allerdings nur in Kombination mit Reden und mit Unterstreichen erreicht. Werden im zweiten Durchgang Wörter herausgeschrieben, schneidet auch dieses Verfahren schlecht ab (Abb. 5). 


\section{Ergebnisse bei den Verfahren des zweiten Rezeptionsdurchgangs (Abb. 7)}

Die Mädchen an Hauptschulen erzielen signifikant bessere Ergebnisse, wenn sie über den Text reden können. Im Gegensatz dazu sind die Ergebnisse signifikant schlechter, wenn aus dem Text Wörter herausgeschrieben werden müssen. ${ }^{35}$

\begin{tabular}{|c|l|c|c|c|}
\hline $\begin{array}{c}\text { Mittelwerte } \\
\text { Mädchen, } \\
\text { Hauptschule }\end{array}$ & \multicolumn{1}{|c|}{ Reden } & Unterstreichen & Schreiben & Gesamt \\
\hline p-Werte & Reden $>$ Unterstreichen 0,17 n. s. & $2,65(2)$. & 2,67 (3.) \\
$\begin{array}{c}\text { Verfahren im } \\
\text { Vergleich }\end{array}$ & $\begin{array}{l}\text { Reden }>\text { Schreiben } 0,03^{*} \\
\text { Unterstreichen }>\text { Schreiben } 0,38 \mathrm{n} \text {. s. }\end{array}$ \\
\hline
\end{tabular}

\section{Abb. 7: Verfahren des zweiten Rezeptionsdurchgangs bei Mädchen an der Hauptschule}

n. s.: nicht signifikant $*$ : signifikant, wenn $\mathrm{p} \leq 0,05$.

\section{Interpretation}

Da im ersten Rezeptionsdurchgang nur das Aktivieren von Vorwissen insgesamt signifikant bessere Ergebnisse aufweist, lenkt sich das Augenmerk der Interpretation auf dieses Verfahren und seine Kombinationen. Der erfolgreiche Einsatz von Aktivieren von Vorwissen wurde bereits im vorigen Kapitel mit der besseren Fähigkeit der Hauptschülerinnen gegenüber den Hauptschülern erklärt, mentale Modelle der Textrepräsentation mit Hilfe von Strategien aufzubauen, die den Top-down-Prozess beim Leseverstehen unterstützen. Diese Fähigkeit hat sich in der Grundschul-Studie abgezeichnet. In der 4. Klasse wurde deutlich, dass das Aktivieren von Vorwissen dann erfolgreich ist, wenn im zweiten Rezeptionsdurchgang "gleichartige", d.h. ebenfalls kommunikative Verfahren kombiniert werden $^{36}$ (Grütz 2004: 40f.). Dieses Ergebnis wird in der Hauptschule bestätigt: M8 Aktivierung von Vorwissen und Reden schneidet signifikant am besten ab. ${ }^{37}$

In Kombination mit Verfahren, die eher der Unterstützung des Bottom-up-Prozesses angehören (Herausschreiben von Wörtern und Unterstreichen), hat in der Grundschul-Studie das Aktivieren von Vorwissen zu schlechten Leseleistungen geführt. Daraus wurde geschlossen, dass es unter den Mädchen bestimmte Lesertypen gibt, die in ihrem Rezeptionsverhalten entweder top-down- oder bottom-up-gesteuert sind (ausführlich hierzu

\footnotetext{
$35 \mathrm{Zu}$ beachten ist jedoch, dass es keinen signifikanten Unterschied macht, wenn beim Rezipieren des Textes die Wahl ist zwischen Reden und Unterstreichen oder zwischen dem Unterstreichen und dem Herausschreiben von Wörtern. Dennoch unterscheiden sich die Mittelwerte deutlich voneinander, was Tendenzen angibt (Abb. 7). Beim Vergleich der einzelnen Methodenkombinationen (Abb. 5) sind bei den Verfahren des zweiten Rezeptionsdurchgangs zunächst keine Gesetzmäßigkeiten zu erkennen. Reden, Unterstreichen und Schreiben sind gleichmäßig auf allen Rängen vertreten. Zu dem deutlichen Ergebnis kommt man durch die gruppierten Verfahren (Abb. 7).

36 Es sei nochmals angemerkt, dass das Vorwissen im Unterrichtsgespräch aktiviert wird.

${ }^{37}$ Vgl. die Signifikanztabelle in Grütz/Belgrad/Pfaff (2004): Forschungsbericht zur Sekundarstufenstudie:111
} 
Grütz 2004: 40f.). Dieses Ergebnis wird in der Hauptschule nur z.T. bestätigt: In der Tat führt die Kombination mit Schreiben (M9) zu einem hochsignifikant schlechteren Ergebnis. Jedoch führt die Kombination mit dem Unterstreichen (M7) zu dem zweitbesten Ergebnis.

In der Sekundarstufen-Studie 7. Klasse kann die These von den "Lesertypen" daher nicht mehr in dieser Deutlichkeit aufrechterhalten werden. ${ }^{38}$ Diese Entwicklung ist insofern nicht verwunderlich, als sich beim Lesenlernen bzw. beim Leseentwicklungsprozess mit der Zeit die bevorzugten Strategien ändern (Valtin 1994: 73). Zu beachten ist auch die Wirksamkeit schulischer Lernprozesse. Eine mögliche Erklärung ist, dass das Unterstreichen in der 7. Klasse - im Gegensatz zur 4. Klasse - eingeübt wurde, aber nicht von allen sicher beherrscht wird.

Aus den Ergebnissen lässt sich die Relevanz der Strategie Aktivieren von Vorwissen ablesen. Diese Strategie findet im zweiten Rezeptionsdurchgang ihre Entsprechung im Verfahren Reden über den Text, das insgesamt am besten, gegenüber dem Schreiben sogar signifikant am besten abschneidet. Damit wird deutlich, dass bei Mädchen in der Hauptschule vor allem kommunikative Strategien Erfolg haben. Das Schreiben führt in der Hauptschule bei Mädchen - genauso wie bei Jungen - zu schlechten Ergebnissen beim Verstehen von Sachtexten.

Zusammenfassend ist festzuhalten: Mädchen in der Hauptschule verfügen besser als Jungen über die Fähigkeit, in der Kognition verankerte Konzepte und Relationen für das Leseverstehen nutzbar zu machen, indem sie diese in der Prä-Lesephase aktivieren und in der Lage sind, die neu einfließenden Informationen mit vorhandenen Inhalten zu verknüpfen. Daher eignet sich für sie die Strategie des Aktivierens von Vorwissen, insbesondere dann, wenn im zweiten Rezeptionsdurchgang über den Text geredet wird, aber auch, wenn Wörter oder Gruppen von Wörtern unterstrichen werden.

\subsection{Ergebnisse bei den Jungen in der Hauptschule}

\section{Ergebnisse bei den Methodenkombinationen (Abb. 8)}

Die besten Ergebnisse erzielen Jungen in der Hauptschule bei allen Methodenkombinationen mit Reden im zweiten Rezeptionsdurchgang: M2 Stillesen und Reden, M8 Aktivierung von Vorwissen und Reden und M5 Vorlesen und Reden. Diese drei Methodenkombinationen liegen im Durchschnittswert gleichwertig an der Spitze. Dennoch gibt es zwischen den Methodenkombinationen der Ränge 1 bis 8 keine signifikanten Unterschiede. Das schlechteste Ergebnis bei den Jungen ist allerdings signifikant: die Methodenkombination M9 Aktivierung von Vorwissen und Schreiben.

\footnotetext{
38 Dafür spräche zwar weiterhin das gute Abschneiden der "gleichartigen" Methodenkombination Aktivierung von Vorwissen und Reden (M8) sowie das schlechte Ergebnis der "verschiedenartigen" Methodenkombination Aktivierung von Vorwissen und Schreiben (M9); es widerspricht hingegen das gute Abschneiden der ebenso "verschiedenartigen" Methodenkombination M7 Aktivierung von Vorwissen und Unterstreichen auf Rang 2; in der Grundschul-Studie 4. Klasse hatte diese Methodenkombination den letzten Platz eingenommen.
} 


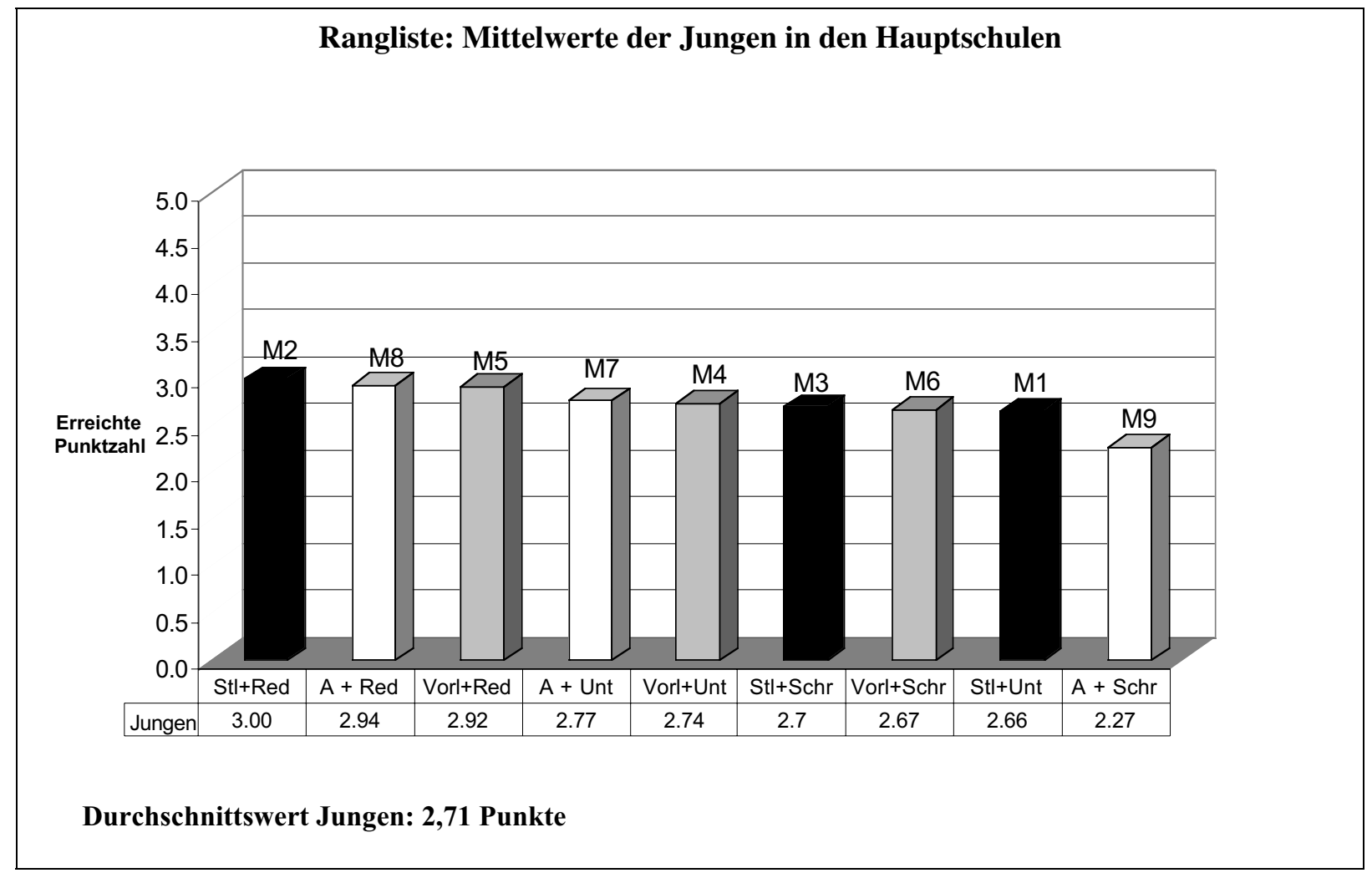

Abb. 8: $\quad$ Rangfolge der Methodenkombinationen bei Jungen in der Hauptschule 7. Klasse

\section{Ergebnisse bei den Verfahren des ersten Rezeptionsdurchgangs (Abb. 9)}

Hauptschüler schneiden tendenziell am besten ab, wenn der Text vorgelesen wird oder wenn sie den Text still lesen. Allerdings weisen die Verfahren des ersten Rezeptionsdurchgangs keine signifikanten Unterschiede auf. ${ }^{39}$ Es ist also weitgehend gleichgültig, mit welchem Verfahren die Jungen in der Hauptschule den Text im ersten Durchgang rezipieren.

\begin{tabular}{|c|c|c|c|c|}
\hline $\begin{array}{l}\text { Mittelwerte } \\
\text { Jungen } \\
\text { Hauptschule }\end{array}$ & Vorlesen & $\begin{array}{l}\text { Stilllesen } \\
2,74 \\
\end{array}$ & Aktiv. v. Vorwissen & Gesamt \\
\hline $\begin{array}{l}\text { p-Werte } \\
\text { Verfahren im } \\
\text { Vergleich }\end{array}$ & \multicolumn{4}{|c|}{$\begin{array}{l}\text { Vorlesen - Stilllesen } 0,62 \mathrm{n} . \mathrm{s} . \\
\text { Vorlesen - Aktivieren von Vorwissen } 0,08 \mathrm{n} . \mathrm{s} . \\
\text { Stilllesen-Aktivieren von Vorwissen } 0,27 \mathrm{n} . \mathrm{s} .\end{array}$} \\
\hline
\end{tabular}

Abb. 9: Verfahren des ersten Rezeptionsdurchgangs bei Jungen an der Hauptschule

n. s.: nicht signifikant $\quad *$ : signifikant $(\mathrm{p} \leq 0,05)$.

39 Zur Vermeidung von Missverständnissen ist darauf hinzuweisen, dass trotz der Gleichrangigkeit der Verfahren

des ersten Rezeptionsdurchgangs die Jungen bei den Verfahren des Stillesens und des Vorlesens bessere Ergebnisse aufweisen als die Mädchen. 


\section{Ergebnisse bei den Verfahren des zweiten Rezeptionsdurchgangs (Abb. 10)}

Hauptschüler präferieren eindeutig das Reden über den Text als zweiten Rezeptionsdurchgang. Kombinationen mit Reden sind signifikant besser als Kombinationen mit Unterstreichen und hochsignifikant besser als Kombinationen mit Herausschreiben von Wörtern.

\begin{tabular}{|c|l|l|c|c|}
\hline $\begin{array}{c}\text { Mittelwerte } \\
\text { Jungen } \\
\text { Hauptschule }\end{array}$ & \multicolumn{1}{|c|}{$\begin{array}{c}\text { Reden } \\
\text { 2,94 (1.) }\end{array}$} & $\begin{array}{c}\text { Unterstreichen } \\
2,73(2 .)\end{array}$ & $\begin{array}{c}\text { Schreiben } \\
2,51(3 .)\end{array}$ & 2,71 \\
\hline p-Werte & $\begin{array}{l}\text { Reden }>\text { Unterstreichen } 0,02^{*} \\
\text { Reden }>\text { Schreiben } 0,00^{* *} \\
\text { Verfahren im } \\
\text { Vergleich }\end{array}$ & $\begin{array}{l} \\
\text { Unterstreichen }>\text { Schreiben } 0,01^{*}\end{array}$ \\
\hline
\end{tabular}

Abb. 10: Verfahren des zweiten Rezeptionsdurchgangs bei Jungen an der Hauptschule

$*$ : signifikant $(\mathrm{p} \leq 0,05) . \quad * *$ : hochsignifikant $(\mathrm{p} \leq 0,00)$.

Das Unterstreichen von Textstellen führt zu Ergebnissen im Mittelfeld, unabhängig davon, mit welcher Methode es im ersten Rezeptionsdurchgang kombiniert wird. Es ist signifikant besser als Schreiben und signifikant schlechter als Reden.

Schreiben führt zu den signifikant schlechtesten Ergebnissen im Test: Es ist signifikant schlechter als Unterstreichen und hochsignifikant schlechter als Reden. Beim Vergleich der einzelnen Methodenkombination (Abb. 8) zeigt sich: Bei Jungen führt das Schreiben in Kombination mit dem Aktivieren von Vorwissen (M9) zum schlechtesten Ergebnis, mit den beiden anderen Verfahren zu Ergebnissen im Mittelfeld.

Vor dem Hintergrund der Grundschul-Studie 4. Klasse (Belgrad/Grütz/Pfaff 2003) sind die Ergebnisse der Sekundarstufen-Studie 7. Klasse bei den Jungen in dreierlei Hinsicht überraschend:

- Das stille Durchlesen eines Textes, das in der Grundschul-Studie 4. Klasse eindeutig das beste Verfahren war, ist bei den Jungen nicht mehr als die erfolgreiche Lesestrategie zu erkennen. In der Sekundarstufe sind im ersten Rezeptionsdurchgang die Verfahren austauschbar. ${ }^{40}$

- Das Reden über den Text, das erfolgreichste Verfahren im zweiten Rezeptionsdurchgang bei Jungen in der Hauptschule, schnitt in der 4. Klasse überwiegend schlecht ab.

- Im Gegensatz zur Studie in der Grundschule sind in der Sekundarstufenstudie 7. Klasse die Leseleistungen bei den einzelnen Methodenkombinationen ausgeglichener. ${ }^{41}$

\footnotetext{
${ }^{40}$ Im Vergleich mit den Mädchen schneiden die Jungen aber mit Stilllesen signifikant besser ab.

${ }^{41}$ Einzig der Mittelwert der Methodenkombination M9 Aktivierung von Vorwissen und Schreiben weicht als schlechtestes Ergebnis signifikant von den anderen Mittelwerten ab.
} 


\section{Interpretation}

In der Grundschul-Studie 4. Klasse wurde das gute Abschneiden des Stilllesen sei den Jungen kognitiv mit der Präferenz der Jungen für Bottom-up-Prozesse beim Leseverstehen begründet und psycho-sozial als Methode, bei der sich die Jungen auf den Text konzentrieren können, ohne von anderen gestört zu werden. Diese Interpretationsansätze werden durch das Ergebnis der hier vorliegenden Studie, das divergente Abschneiden des Stilllesens, nicht ungültig. Zu beachten ist die unterschiedliche Schülerstruktur in der Grundschule und in der Sekundarstufe: In der Grundschule sind Schülerinnen und Schüler aller Intelligenzgrade und aus allen Bildungsschichten vertreten. Der Mittelwert einer Methodenkombination kann sich daher aus einer intelligenz- und bildungsmäßig breit gestreuten Schülerschaft ergeben. ${ }^{42}$

In der Sekundarstufe werden in der 5. Klasse die leistungsschwachen und leistungsstarken Schülerinnen und Schüler getrennt und auf die Schultypen Hauptschule, Realschule und Gymnasium verteilt. Dass es sich in der Hauptschule überwiegend um leistungsschwache Schülerinnen und Schüler handelt, belegt das Durchschnittsergebnis der SekundarstufenStudie 7. Klasse: Während die Schülerinnen und Schüler in der Hauptschule einen durchschnittlichen Mittelwert von 2,69 Punkten erreichten, erzielten die Schülerinnen und Schüler in der Realschule einen Mittelwert von 3,60 Punkten, im Gymnasium ${ }^{43}$ von 4,44 Punkten. Bei insgesamt fünf zu erreichenden Punkten ist der Unterschied von jeweils fast einem Punkt erheblich. Angesichts der großen Unterschiede in der Leseleistung ist es auch nahe liegend, dass unterschiedliche Methoden präferiert werden.

Das eindeutig gute Abschneiden des Redens bei Hauptschülern ruft Unsicherheiten in der Interpretation hervor. Zwar ist aus kognitionspsychologischer Sicht davon auszugehen, dass Jungen dieser Altersstufe und mit einer vergleichbar geringen Lesekompetenz die elaborative Methode des Redens bevorzugen, weil ihnen in Kooperation mit anderen Rezipienten der Aufbau eines mentalen Modells gelingt. Die große Bedeutung des Redens für Jungen in der Hauptschule widerspricht aber Befunden vieler Studien (z. B. Rüegg 1995, van Alphen 1996, Fuchs 2001), in denen deutlich wird, dass Jungen das Unterrichtsgespräch eher für die Selbstdarstellung als für die inhaltliche Beteiligung am Unterricht nutzen. Eine mögliche Antwort auf diesen Widerspruch könnte sein: Angesichts der allgemein schlechten Ergebnisse der Hauptschülerinnen und -schüler in den Leseleistungen, die auf kognitive Defizite und auf einen Mangel an Konzentrationsfähigkeit zurückgeführt werden können, schneiden Verfahren, die auf selbstständiges Arbeiten setzen, schlecht ab. Das Reden über den Text gewährt von Seiten der Lehrkraft Unterstützung beim Verstehen; es schneidet daher trotz der für Jungen als problematisch zu betrachtenden Methode noch am besten von allen anderen Verfahren $a b$.

Allerdings ist bei diesem Interpretationsansatz zu fragen, warum dann nicht auch die Methode Aktivieren von Vorwissen, die in der vorliegenden Studie durch ein Unterrichtsgespräch realisiert wurde, präferiert wird. Eine Antwort könnte sein: Beim Aktivieren von Vorwissen

\footnotetext{
42 Die Faktoren Intelligenz und Bildungsgrad/soziale Herkunft wurden in der Grundschul-Studie nicht erhoben.

${ }^{43}$ Noch unveröffentlichte Parallelstudie (Grütz/Zmaila 2006).
} 
spielt der Text bzw. die Arbeit an diesem noch keine Rolle. Die Strategie Aktivieren von Vorwissen knüpft bei metakognitiven Fähigkeiten an, die bei den Jungen an der Hauptschule womöglich (noch) nicht entwickelt oder besonders ausgeprägt sind. Bei der Strategie Reden über den Text hingegen hat ein datengeleiteter Verstehensprozess bereits eingesetzt. Ein propositionales Textmodell wurde aufgebaut, an das nun im Unterrichtsgespräch angeknüpft werden kann. Die noch lückenhafte Textrepräsentation wird mit weiteren Informationen angereichert und akzentuiert. Dieser Verstehensprozess kann so weit gehen, dass der Text hauptsächlich durch die Informationen, die die Lehrkraft und andere Schülerinnen und Schüler im Unterrichtsgespräch geben, rezipiert und verstanden wird. Diese Theorie müsste durch weitere Untersuchungen geprüft werden.

Zusammenfassend ist festzuhalten: Bei Jungen ist es weitgehend gleichgültig, mit welchem Verfahren die erste Textbegegnung stattfindet. Ausschlaggebend ist, dass im zweiten Rezeptionsdurchgang über den Text geredet wird. Mit diesem Verfahren werden die besten Ergebnisse erzielt. Warum das Reden so eindeutig zum Verstehenserfolg führt, kann allerdings nicht hinreichend interpretiert werden. Schreiben führt zu den signifikant schlechtesten Ergebnissen. Dies ist damit zu erklären, dass in bildungsfernen Elternhäusern das Schreiben nicht zu den geübten Kulturtechniken gehört.

\subsection{Zusammenfassung: Leseleistung und Rezeptionsstrategien bei Mädchen und Jungen in der Hauptschule}

In der Hauptschule 7. Klasse kommt es bei Mädchen und Jungen zu folgenden Ergebnissen beim Verstehen von Sachtexten:

- In der Leseleistung liegt im Durchschnitt kein Unterschied zwischen Mädchen und Jungen vor. Sie präferieren allerdings unterschiedliche Rezeptionsstrategien.

- Verfahren des ersten Rezeptionsdurchgangs:

Bei Jungen ist es gleichgültig, mit welchem Verfahren sie den Text zuerst rezipieren; es gibt keinen signifikanten Unterschied. Dennoch schneiden Jungen signifikant besser ab als Mädchen, wenn der Text zuerst still gelesen wird oder wenn die Lehrkraft ihn vorliest. - Mädchen erzielen tendenziell ein besseres Ergebnis als Jungen, wenn vor dem Lesen das Vorwissen aktiviert wird.

- Verfahren des zweiten Rezeptionsdurchgangs:

Mädchen und Jungen verstehen Sachtexte signifikant am besten, wenn im zweiten Durchgang über den Text geredet wird. Zu einem mittleren Erfolg kommt es, wenn Textstellen unterstrichen werden; am schlechtesten werden Sachtexte verstanden, wenn Wörter herauszuschreiben sind. Während diese Unterschiede bei den Jungen signifikant sind, erweisen sich die Ergebnisse bei den Mädchen als ausgeglichener. Bei Mädchen ist nur der Unterschied zwischen Reden und Schreiben signifikant. 
- Methodenkombination mit bestem Ergebnis:

Mädchen erzielen ihr bestes Ergebnis mit der Methodenkombination M8 Aktivierung von Vorwissen und Reden. Die Jungen erzielen damit ihr zweitbestes Ergebnis. Diese Kombination scheint als besonders verstehensfördernd bei der Rezeption von Sachtexten zu sein. Jungen erzielen ihr bestes Ergebnis mit der Methodenkombination M2 Stilllesen und Reden. Diese Kombination führt bei den Mädchen nur zu einem Ergebnis im Mittelfeld.

- Methodenkombination mit schlechtestem Ergebnis:

Mädchen weisen bei der Methodenkombination M1 Stilllesen und Unterstreichen ihr schlechtestes Ergebnis auf. Diese Methodenkombination liegt bei den Jungen im Mittelfeld. Jungen weisen bei der Methodenkombination M9 Aktivierung von Vorwissen und Schreiben ihr schlechtestes Ergebnis auf. Diese Kombination schneidet bei den Mädchen ebenfalls schlecht ab. 


\section{Rezeptionsstrategien von Mädchen und Jungen in der Realschule}

\subsection{Die wichtigsten Ergebnisse im Überblick}

1. Im 7. Schuljahr Realschule gibt es in der Leseleistung insgesamt keinen Unterschied zwischen Mädchen und Jungen beim Verstehen von Sachtexten.

2. Mädchen schneiden in der Realschule am besten ab, wenn sie im ersten Rezeptionsdurchgang einen Text still lesen können. Sie sind bei dieser Methode den Jungen überlegen. Die Verfahren des zweiten Rezeptionsdurchgangs sind bei den Realschülerinnen für das Verstehen von Sachtexten nicht Ausschlag gebend. Im Vergleich mit den Jungen sind sie beim Herausschreiben von Wörtern tendenziell besser. Die Mädchen erzielen mit drei Methodenkombinationen die besten Ergebnisse:

Stilllesen + Schreiben (M3)

Stilllesen + Reden (M2)

Aktivierung von Vorwissen + Unterstreichen (M7).

3. Jungen schneiden in der Realschule im ersten Rezeptionsdurchgang gleichermaßen gut mit Aktivieren von Vorwissen und mit Stilllesen ab. Im zweiten Rezeptionsdurchgang erzielen sie die besten Ergebnisse, wenn über den Text geredet wird oder wenn Wörter unterstrichen werden. Müssen sie aus dem Text Wörter herausschreiben, kommen Jungen zu ihrem signifikant schlechtesten Ergebnis.

Die Jungen erzielen ihr bestes Ergebnisse mit der Methodenkombination Aktivierung von Vorwissen + Unterstreichen (M7).

4. Mädchen und Jungen kommen an der Realschule mit der gleichen Methodenkombination zum besten und zum schlechtesten Ergebnis: Aktivierung von Vorwissen + Unterstreichen (M7) führt zu sehr guten Leseleistungen, Vorlesen und Schreiben (M6) zum schlechtesten Ergebnis.

\section{2 Ergebnisse bei Mädchen und Jungen an Realschulen im Vergleich}

Bei den Ergebnissen von Mädchen und Jungen in der Realschule ergeben sich zwei grundlegende Befunde:

\section{Befund: Mädchen und Jungen erzielen gleiche Leseleistungen.}

Beim Verstehen von Sachtexten weisen Mädchen und Jungen in der Realschule keinen Unterschied in der Leseleistung auf. ${ }^{44}$ Beide Geschlechter erzielen einen gleichen Durchschnittswert aller Methodenkombinationen (3,6 Punkte). Auch bei den

\footnotetext{
${ }^{44}$ Zur Interpretation dieses Befundes siehe weiter oben Kapitel 2.1.
} 

bei Mädchen und Jungen in der Sekundarstufe I

Methodenkombinationen lassen sich - im Gegensatz zur Hauptschule - nur geringfügige Unterschiede feststellen (Abb. 11). Lediglich eine Methodenkombination weist einen deutlichen Unterschied auf: M3 Still lesen und Schreiben. Hier schneiden die Mädchen signifikant besser ab als die Jungen (vgl. Tab. 6 Anhang).

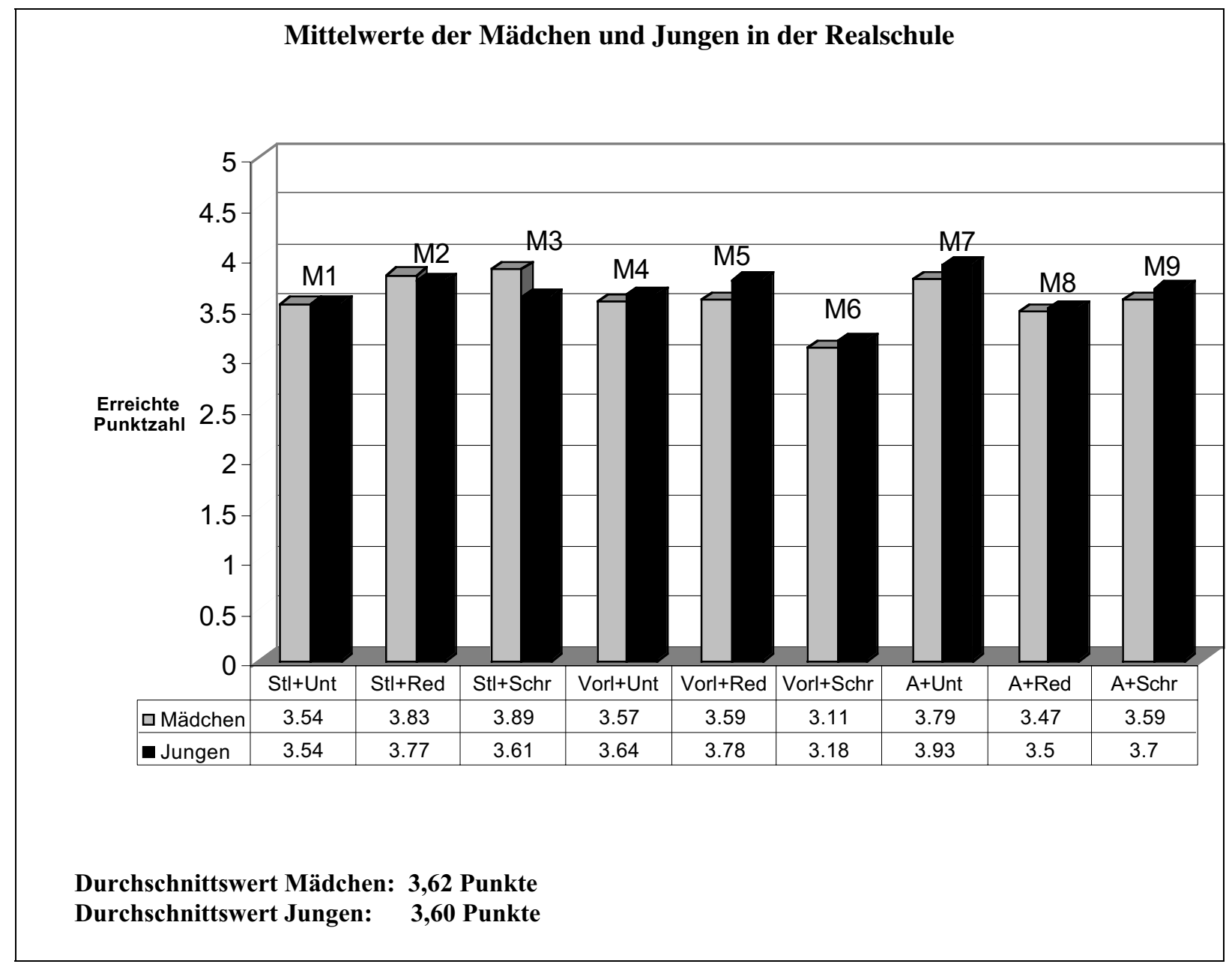

Abb. 11: Mittelwerte der Mädchen und Jungen in der Realschule 7. Klasse

2. Befund: $\quad$ Mädchen sind den Jungen in der Realschule beim stillen Lesen überlegen. Ansonsten präferieren beide Geschlechter weitgehend gleiche Rezeptionsstrategien.

\section{Ergebnisse bei den Methodenkombinationen (Abb. 11)}

Beim Vergleich der Methodenkombinationen zeigt sich, dass bei zwei Kombinationen beide Geschlechter ein sehr gutes bzw. gutes Ergebnis erzielen:

M7 Aktivierung von Vorwissen und Unterstreichen (Jungen Rang 1, Mädchen Rang 3), M2 Stilllesen und Reden (Mädchen Rang 2, Jungen Rang 3) (vgl. auch Abb. 14 und Abb. 17) 


\section{Ergebnisse bei Verfahren des ersten Rezeptionsdurchgangs (Abb. 12)}

Im ersten Rezeptionsdurchgang sind die Mädchen den Jungen beim Stilllesen eines Sachtextes überlegen (Abb. 12). Dieses Ergebnis ist fast signifikant. Bei den anderen Verfahren gibt es zwischen den Geschlechtern insgesamt keine signifikanten Unterschiede. Beide Geschlechter erhalten ihr schlechtestes Ergebnis, wenn der Text von der Lehrkraft vorgelesen wird.

\section{Ergebnisse bei den Verfahren des zweiten Rezeptionsdurchgangs (Abb. 13)}

Im zweiten Rezeptionsdurchgang ergeben sich keine signifikanten Unterschiede zwischen Mädchen und Jungen in der Realschule. Beide Geschlechter erzielen gleiche Werte beim Reden und beim Unterstreichen. Wenn aus dem Text Wörter herauszuschreiben sind, schneiden die Mädchen im Mittelwert besser ab als die Jungen. Dieser Unterschied ist aber nicht signifikant.

\begin{tabular}{|c|c|c|c|c|}
\hline $\begin{array}{c}\text { 1. Rezeptions- } \\
\text { durchgang }\end{array}$ & Stilllesen & $\begin{array}{c}\text { Aktivieren von } \\
\text { Vorwissen } \\
\end{array}$ & Vorlesen & Bei Stilllesen \\
\hline $\begin{array}{l}\text { Mittelwert } \\
\text { Mädchen, } \\
\text { Realschule }\end{array}$ & 3,77 (1.) & 3,58 (2.) & 3,45 & $\begin{array}{l}\text { Unterschied } \\
\text { fast signifikant }\end{array}$ \\
\hline $\begin{array}{l}\text { Mittelwert } \\
\text { Jungen, Realschule }\end{array}$ & 3,63 (1.) & 3,66 (1.) & 3,51 (3.) & $\begin{array}{l}\text { keine } \\
\text { signifikanten } \\
\text { Unterschiede }\end{array}$ \\
\hline $\begin{array}{l}\text { p-Wert } \\
\text { Mädchen - Jungen }\end{array}$ & $\begin{array}{c}\mathbf{0 , 0 6} \text { fast } * \\
\text { (Mädchen besser) }\end{array}$ & 0,36 n.s. & $\mathbf{0 , 5 1}$ n. s. & $\begin{array}{l}\text { bei Aktivieren } \\
\text { von Vorwissen } \\
\text { und Vorlesen }\end{array}$ \\
\hline
\end{tabular}

Abb. 12: Vergleich der Verfahren des ersten Rezeptionsdurchgangs nach Geschlecht

n. s.: nicht signifikant $*$ : signifikant $(\mathrm{p} \leq 0,05)$

\begin{tabular}{|l|c|c|c|l|}
\hline $\begin{array}{l}\text { 2. Rezeptions- } \\
\text { durchgang }\end{array}$ & Reden & Unterstreichen & Schreiben & \\
\cline { 1 - 3 } $\begin{array}{l}\text { Mittelwert } \\
\text { Mädchen, Realschule }\end{array}$ & 3,65 & 3,60 & 3,60 & keine \\
signifikanten \\
$\begin{array}{l}\text { Mittelwert } \\
\text { Jungen, Realschule }\end{array}$ & 3,67 & 3,65 & 3,49 & Unterschiede \\
\cline { 1 - 3 } $\begin{array}{l}\text { p-Wert } \\
\text { Mädchen - Jungen }\end{array}$ & $\mathbf{0 , 7 8}$ n. s. & $\mathbf{0 , 5 2}$ n. s. & $\mathbf{0 , 1 4}$ n. s. & \\
\hline
\end{tabular}

Abb. 13: Vergleich der Verfahren des zweiten Rezeptionsdurchgangs nach Geschlecht

n. s.: nicht signifikant 


\section{Interpretation}

Das signifikant bessere Abschneiden der Mädchen bei M3 Still lesen und Schreiben ist zunächst eine Bestätigung der Befunde von Richter/May/Brügelmann (1994), die eine Überlegenheit der Mädchen beim Schriftspracherwerb zwischen der 1. und der 9. Klasse feststellen (1994: 157f). Ein generell besseres Abschneiden beim Schreiben lässt sich in der vorliegenden Studie für die Realschülerinnen aber nicht belegen: Bei M6 Vorlesen und Schreiben und M9 Aktivierung von Vorwissen und Schreiben schneiden die Jungen geringfügig besser ab. Vermutlich ist für das Leseverstehen der Effekt des ersten Rezeptionsdurchgangs bei beiden Geschlechtern größer als der des zweiten Rezeptionsdurchgangs. ${ }^{45}$

\footnotetext{
45 Betrachtet man allerdings nicht die Geschlechter im Vergleich, sondern jeweils getrennt, ergeben sich für das Verfahren Schreiben differenziertere Aussagen. Siehe die beiden folgenden Kapitel.
} 


\subsection{Ergebnisse bei den Mädchen in der Realschule}

\section{Ergebnisse bei den Methodenkombinationen (Abb. 14)}

Bei den Mädchen führen drei Methodenkombinationen zu den signifikant besten Ergebnissen:

Stilllesen + Schreiben (M3)

Stilllesen + Reden (M2)

Aktivierung von Vorwissen + Unterstreichen (M7).

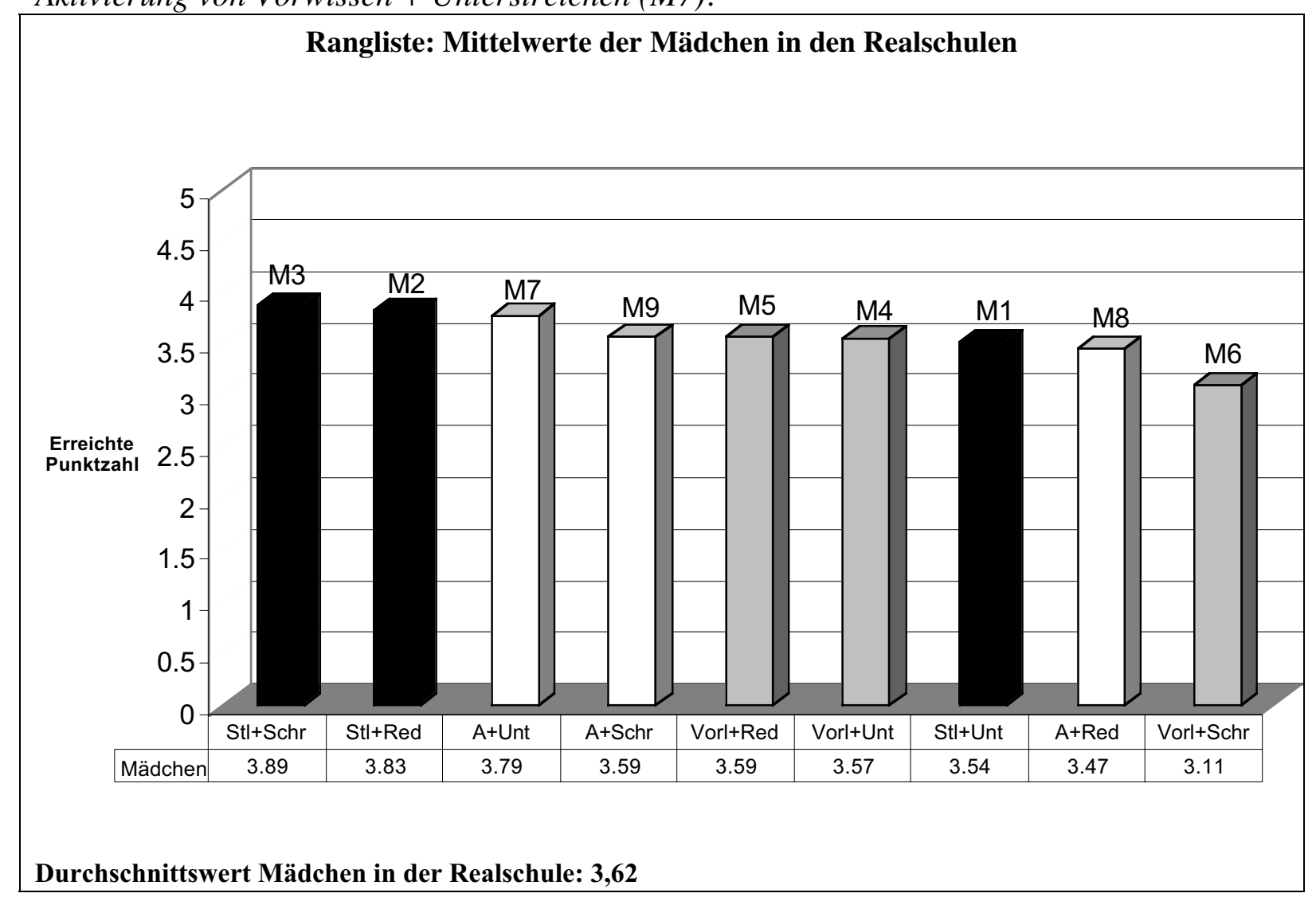

Abb. 14: Rangliste der Methodenkombinationen bei Mädchen in der Realschule 7. Klasse

Realschülerinnen verstehen Sachtexte dann gut, wenn sie den Text zuerst still lesen und anschließend aus dem Text Wörter herausschreiben oder über den Text reden. Sachtexte werden von Mädchen ebenso gut verstanden, wenn zuerst im Unterrichtsgespräch Vorwissen zum Thema aktiviert wird und anschließend im Text Wörter unterstrichen werden.

Das signifikant schlechteste Ergebnis bei den Mädchen bewirkt die Methodenkombination Vorlesen und Schreiben (M6), d.h. der Text wird zuerst von der Lehrkraft vorgelesen, die Realschülerinnen schreiben anschließend aus dem Text Wörter heraus. Alle anderen Methodenkombinationen liegen gleichwertig im Mittelfeld.

Ergebnisse bei den Verfahren des ersten Rezeptionsdurchgangs (Abb. 15)

Im ersten Rezeptionsdurchgang hat sich bei den Mädchen in der Realschule eine signifikante Präferenz für das Stilllesen ergeben: Es ist hochsignifikant besser als Vorlesen und signifikant 
besser als Aktivieren von Vorwissen. Realschülerinnen verstehen Sachtexte also dann am besten, wenn sie diese still durchlesen können. Hingegen ist es bei ihnen weitgehend gleichgültig, ob der Text zuerst von der Lehrkraft vorgelesen wird oder ob vor dem Lesen Vorwissen aktiviert wird.

\begin{tabular}{|l|c|c|c|c|}
\hline 1. Rezeptionsdurchgang & Stillesen & $\begin{array}{c}\text { Aktivierung von } \\
\text { Vorwissen }\end{array}$ & Vorlesen & Gesamt \\
\hline $\begin{array}{l}\text { Mittelwert } \\
\text { Mädchen, Realschule }\end{array}$ & $3,77(1)$. & $3,58(2)$. & $3,45(3)$. & 3,62 \\
\hline \multicolumn{1}{|c|}{ p-Werte } & $\begin{array}{l}\text { Stillesen }>\text { Aktivieren von Vorwissen } 0,01^{*} \\
\text { Stilllesen }>\text { Vorlesen } 0,00^{* *} \\
\text { Aktivieren von Vorwissen }>\text { Vorlesen } 0,10 \mathrm{n} \text {. s. }\end{array}$ \\
\hline
\end{tabular}

Abb. 15: Vergleich der Verfahren des ersten Rezeptionsdurchgangs bei Mädchen an Realschulen

n. s.: nicht signifikant $*$ signifikant $(\mathrm{p} \leq 0,05) * *$ hochsignifikant $(\mathrm{p} \leq 0,00)$

\section{Ergebnisse bei den Verfahren des zweiten Rezeptionsdurchgangs (Abb. 16)}

Im zweiten Rezeptionsdurchgang gibt es bei den Mädchen in der Realschule keinerlei Unterschiede. Es ist gleichgültig, ob beim zweiten Rezipieren Textstellen unterstrichen oder Wörter herausgeschrieben werden oder ob über den Text geredet wird.

\begin{tabular}{|l|c|c|c|c|}
\hline 2. Rezeptionsdurchgang & Reden & Unterstreichen & Schreiben & Gesamt \\
\hline $\begin{array}{l}\text { Mittelwert } \\
\text { Mädchen, Realschule }\end{array}$ & 3,65 & 3,60 & 3,60 & 3,62 \\
\hline \multicolumn{1}{|c|}{ p-Werte } & $\begin{array}{l}\text { Reden }>\text { Unterstreichen } 0,51 \text { n. s. } \\
\text { Reden }>\text { Schreiben } 0,57 \text { n. s. } \\
\text { Unterstreichen - Schreiben } 0,92 \text { n. s. }\end{array}$ & $\begin{array}{l}\text { keine } \\
\text { signifikanten } \\
\text { Unterschiede }\end{array}$ \\
\hline
\end{tabular}

Abb. 16: Vergleich der Verfahren des zweiten Rezeptionsdurchgangs bei Mädchen an Realschulen

n. s.: nicht signifikant

\section{Interpretation}

Auf den ersten Blick sind bei der Rangfolge der Methodenkombinationen keine Gesetzmäßigkeiten ablesbar. Erst die gruppierten Verfahren geben Aufschluss: Das stille Lesen eines Sachtextes ist bei den Mädchen als erfolgreiche Methode für das Verstehen zu benennen: In Kombination mit dem Herausschreiben von Wörtern und in Kombination mit dem Reden über den Text führt es zu den beiden besten Ergebnissen. In Kombination mit dem Unterstreichen von Wörtern zeigt sich immerhin ein Ergebnis im Mittelfeld. Im Vergleich zu den Ergebnissen der Grundschul-Studie (Belgrad/Grütz/Pfaff 2003) haben die Mädchen in der 
Realschule ihre Präferenz geändert. Zwar wurden auch in der 4. Klasse mit Stilllesen gute Leseleistungen erzielt, die präferierte Methode war jedoch das Aktivieren von Vorwissen.

Dass es bei den Mädchen an der Realschule gleichgültig ist, mit welchem Verfahren der Sachtext im zweiten Durchgang rezipiert wird, kann daran liegen, dass diese Verfahren in der Schule gleichermaßen eingeübt wurden und Mädchen diese Verfahren beherrschen. Es kann aber auch sein, dass bei den Mädchen das Verfahren des ersten Rezeptionsdurchgangs für das Verstehen von Sachtexten ausschlaggebend ist.

\subsection{Ergebnisse bei den Jungen in der Realschule}

\section{Ergebnisse bei den Methodenkombinationen (Abb. 17)}

Beim Vergleich der Methodenkombinationen lassen sich bei den Jungen in der Realschule keine Gesetzmäßigkeiten erkennen. Das beste Ergebnis wird durch die Methodenkombination M7 Aktivierung von Vorwissen und Unterstreichen erreicht. Das signifikant schlechteste Ergebnis wird durch die Methodenkombination M6 Vorlesen und Schreiben herbeigeführt. Alle anderen Methodenkombinationen liegen gleichwertig im Mittelfeld.

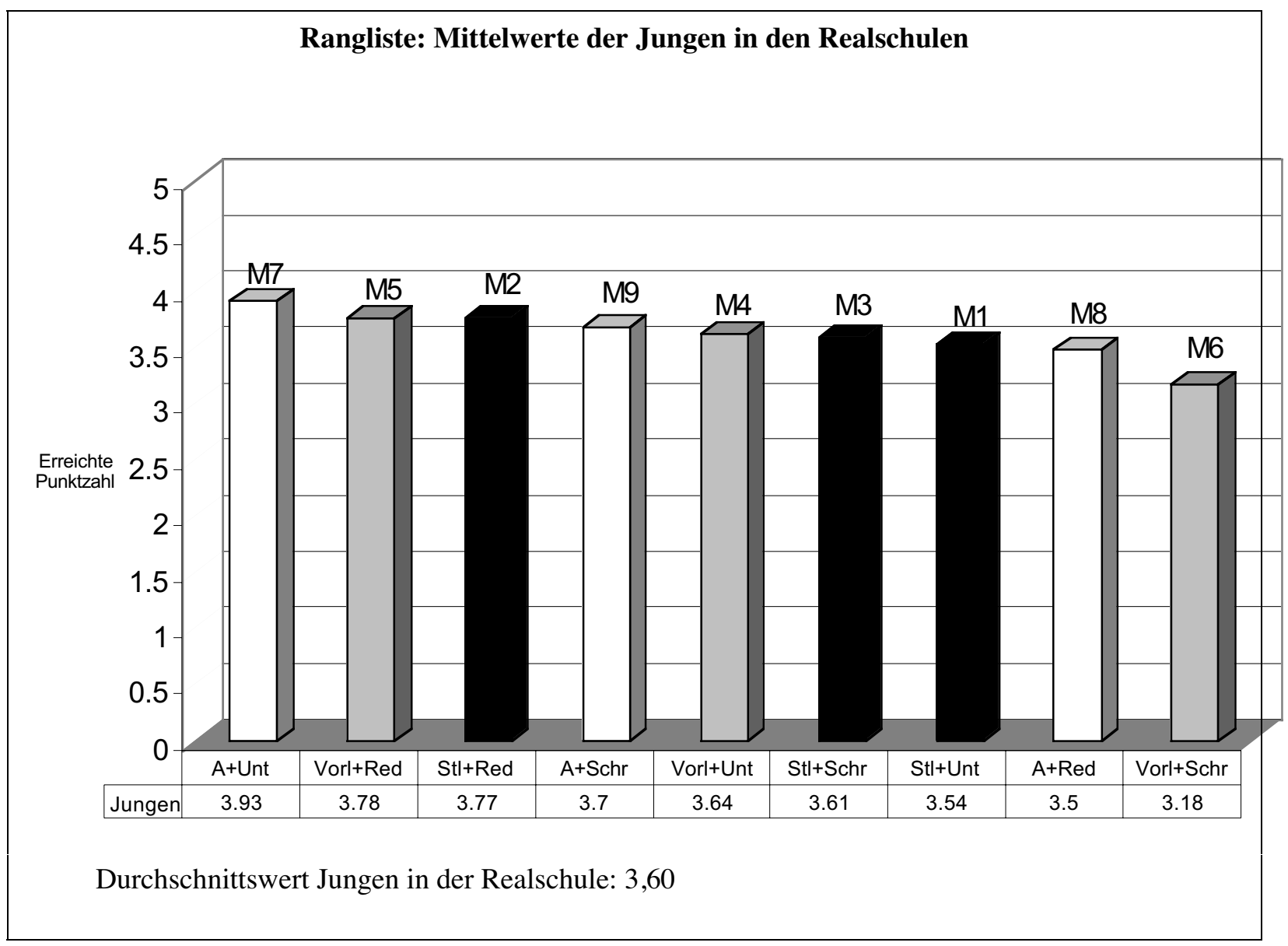

Abb. 17: Rangliste der Methodenkombinationen bei Jungen in der Realschule 7. Klasse 


\section{Ergebnisse bei den Verfahren des ersten Rezeptionsdurchgangs (Abb. 18)}

Im ersten Rezeptionsdurchgang konnte kein Verfahren als besonders günstig oder ungünstig für den Leseerfolg ermittelt werden. Das Stilllesen schneidet genauso gut ab wie das Aktivieren von Vorwissen. Nur das Vorlesen eines Textes hat den schlechtesten Mittelwert. Es ist gegenüber dem Aktivieren von Vorwissen deutlich schlechter. Der Unterschied erreicht fast das statistische Signifikanzniveau.

\section{Ergebnisse bei den Verfahren des zweiten Rezeptionsdurchgangs (Abb. 19)}

Beim zweiten Rezeptionsdurchgang führen bei Jungen in der Realschule das Unterstreichen und das Reden über den Text gleichwertig zum Verstehenserfolg. Das Herausschreiben von Wörtern führt hingegen zum schlechtesten Ergebnis: Im Vergleich zum Reden ist es signifikant schlechter, im Vergleich zum Unterstreichen ist es fast signifikant schlechter (p: 0,06).

\begin{tabular}{|l|c|c|c|c|}
\hline 1. Rezeptionsdurchgang & $\begin{array}{l}\text { Aktivierung von } \\
\text { Vorwissen }\end{array}$ & Stillesen & Vorlesen & Gesamt \\
\hline $\begin{array}{l}\text { Mittelwert } \\
\text { Jungen, Realschule }\end{array}$ & $3,66(1)$. & $3,63(1)$. & 3,51 (3.) & 3,60 \\
\hline p-Werte & $\begin{array}{l}\text { Aktivieren von Vorwissen }>\text { Stilllesen } 0,77 \mathrm{n} \text {. s. } \\
\text { Aktivieren von Vorwissen }>\text { Vorlesen } 0,07 \text { fast * } \\
\text { Stillesen }>\text { Vorlesen } 0,11 \mathrm{n} \text {. s. }\end{array}$ \\
\hline
\end{tabular}

Abb. 18: Vergleich der Verfahren des ersten Rezeptionsdurchgangs bei Jungen an Realschulen

n. s.: nicht signifikant $*$ signifikant $(\mathrm{p} \leq 0,05) \quad * *$ hochsignifikant $(\mathrm{p} \leq 0,00)$

\begin{tabular}{|l|c|c|c|c|}
\hline 2. Rezeptionsdurchgang & Reden & Unterstreichen & Schreiben & Gesamt \\
\hline $\begin{array}{l}\text { Mittelwert } \\
\text { Jungen, Realschule }\end{array}$ & $3,67(1)$. & $3,65(1)$. & $3,49(3)$. & 3,60 \\
\hline \multicolumn{1}{|l|}{ p-Werte } & $\begin{array}{l}\text { Reden - Unterstreichen } 0,79 \text { n. s. } \\
\text { Reden }>\text { Schreiben 0,03* } \\
\text { Unterstreichen > Schreiben 0,06 fast * }\end{array}$ \\
\hline
\end{tabular}

Abb. 19: Vergleich der Verfahren des zweiten Rezeptionsdurchgangs bei Jungen an Realschulen

n. s.: nicht signifikant $*$ signifikant $(\mathrm{p} \leq 0,05) \quad * *$ hochsignifikant $(\mathrm{p} \leq 0,00)$ 


\section{Interpretation}

Im ersten Rezeptionsdurchgang ist es bei Jungen in der Realschule gleichgültig, ob der Text zuerst still gelesen wird oder ob zuerst Vorwissen aktiviert wird. Es ist zu vermuten, dass gleichermaßen Bottom-up-Prozesse und Top-down-Prozesse das Textverstehen beeinflussen. Im Vergleich zur Grundschule hat sich hier eine Änderung ergeben: Das Stilllesen war bei Jungen in der 4. Klasse das Verfahren mit den besten Ergebnissen (Belgrad/Grütz/Pfaff 2003). Aus dem schlechteren Abschneiden der Jungen beim Vorlesen kann man vorsichtig schließen, dass das Vorlesen ein für Sachtexte ungeeignetes Verfahren ist.

Deutlich ist bei den Jungen das schlechte Abschneiden des Herausschreibens von Wörtern. Dieser Befund bestätigt Ergebnisse anderer Studien (Richter/May/Brügelmann 1994: 157f). Vermutlich schreiben Jungen in ihrer Freizeit wenig. Tagebucheinträge, wie bei den Mädchen, finden kaum statt. Das Schreiben wird weniger geübt und behindert offenbar den Verstehensprozess. 


\subsection{Zusammenfassung: Leseleistung und Rezeptionsstrategien bei Mädchen und Jungen in der Realschule}

1. Im 7. Schuljahr Realschule gibt es insgesamt keinen Unterschied zwischen Mädchen und Jungen beim Verstehen von Sachtexten.

2. Bei beiden Geschlechtern gibt es folgende Übereinstimmungen bei den Methodenkombinationen: Mädchen und Jungen an Realschulen erzielen gute Ergebnisse beim Aktivieren von Vorwissen + Unterstreichen (M7) und beim Stilllesen + Reden (M2). Die Methodenkombination Vorlesen + Schreiben (M6) führt bei beiden Geschlechtern zum schlechtesten Ergebnis.

3. Der einzige signifikante Unterschied in der Leseleistung von Mädchen und Jungen tritt bei der Methodenkombination Still lesen + Schreiben (M3) auf. Hier schneiden die Mädchen besonders gut ab; bei den Jungen entspricht das dem Durchschnittswert.

\section{Die Ergebnisse bei den Mädchen an der Realschule}

Drei Methodenkombinationen führen zu den besten Ergebnissen:

Stilllesen + Schreiben (M3)

Stilllesen + Reden (M2)

Aktivierung von Vorwissen + Unterstreichen (M7).

Das mit Abstand schlechteste Ergebnis erhalten die Realschülerinnen mit Vorlesen und Schreiben (M6). Alle anderen Methodenkombinationen liegen im Mittelfeld. Im ersten Rezeptionsdurchgang kann bei den Realschülerinnen das stille Durchlesen eines Sachtextes als generell erfolgreiches Verfahren angesehen werden. Vorlesen schneidet am schlechtesten ab. Im zweiten Rezeptionsdurchgang kommen die Realschülerinnen mit allen drei untersuchten Verfahren - Unterstreichen, Reden über den Text, Herausschreiben von Wörtern - zu gleichen Ergebnissen. Es ist also gleichgültig, mit welchem Verfahren sie den Text beim zweiten Durchgang rezipieren.

\section{Die Ergebnisse bei den Jungen an der Realschule}

Das beste Ergebnisse erzielen die Jungen mit Aktivierung von Vorwissen + Unterstreichen (M7). Das mit Abstand schlechteste Ergebnis wird mit Vorlesen und Schreiben (M6) erreicht. Alle anderen Methodenkombinationen liegen im Mittelfeld. Im ersten Rezeptionsdurchgang erzielen Realschüler mit Aktivieren von Vorwissen und mit dem stillen Durchlesen eines Textes ihre besten Ergebnisse. Vorlesen schneidet am schlechtesten ab. Im zweiten Rezeptionsdurchgang zeigen Kombinationen mit dem Unterstreichen und mit dem Reden gleichwertige Erfolge. Das Herausschreiben von Wörtern schneidet signifikant am schlechtesten ab. 


\section{$5 \quad$ Genderspezifische Rezeptionsstrategien in der Sekundarstufe I - ein Gesamtüberblick}

Beim Vergleich von Mädchen und Jungen sind in der 7. Klasse an Hauptschulen und Realschulen folgende Befunde hervorzuheben:

1. Bei gleichem Bildungsniveau, also innerhalb eines Schultyps, gibt es zwischen Mädchen und Jungen insgesamt keine Unterschiede in der Leseleistung.

In der Hauptschule erreichen von fünf erzielbaren Punkten die Mädchen 2,66 Punkte, die Jungen 2,71 Punkte. Dieser Unterschied ist nicht signifikant.

Die Realschülerinnen und -schüler erreichen im Durchschnitt etwa einen Punkt mehr und schneiden damit signifikant besser ab als die Hauptschülerinnen und -schüler. An der Realschule schneiden Mädchen und Jungen im Ergebnis gleich ab: Mädchen erzielen im Durchschnitt 3,62 Punkte, Jungen 3,60 Punkte.

2. In der Hauptschule unterscheiden sich die Rezeptionsstrategien zwischen Mädchen und Jungen beim ersten Rezeptionsdurchgang: Jungen präferieren Stilllesen und Vorlesen, Mädchen Aktivieren von Vorwissen.

Die Jungen weisen signifikant bessere Ergebnisse als die Mädchen auf, wenn der Text bei der ersten Textbegegnung still durchgelesen wird oder wenn die Lehrkraft ihn vorliest. Die Mädchen weisen wesentlich bessere Ergebnisse als die Jungen auf, wenn vor der ersten Textbegegnung im Unterrichtsgespräch Vorwissen aktiviert wird.

Im zweiten Rezeptionsdurchgang gibt es keine signifikanten Unterschiede zwischen den Geschlechtern: Reden schneidet bei Mädchen und Jungen in der Hauptschule signifikant am besten, Unterstreichen mit mittlerem Erfolg, Schreiben signifikant am schlechtesten ab.

Mädchen und Jungen kommen zum besten Ergebnis, wenn sie nach der ersten Textbegegnung im Unterrichtsgespräch über den Text reden können. Sind in einem Sachtext Wörter zu unterstreichen, kommen beide Geschlechter zu einem mittleren Leseerfolg. Das signifikant schlechteste Ergebnis erzielen Mädchen und Jungen, wenn sie aus dem Text Wörter herausschreiben müssen. Bei den Jungen sind die Unterschiede zwischen den drei Verfahren ausgeprägter als bei Mädchen.

Einen geschlechtsspezifisch unterschiedlichen Zugriff haben Schülerinnen und Schüler an der Hauptschule also nur beim ersten Rezipieren eines Sachtextes. Vermutlich können die Mädchen an der Hauptschule das Aktivieren von Vorwissen lesestrategisch besser als Topdown-Prozess nutzen als Jungen. Bei den Jungen ist es an der Hauptschule offensichtlich wichtig, einen Text zuerst konzentriert in Stillarbeit zu rezipieren und im Bottom-up-Prozess ein propositionales Textmodell aufzubauen. Das stille Lesen ermöglicht eine Textrezeption im individuellen Tempo; es kann nach Bedarf vor- und zurückgelesen werden. Das Vorlesen durch die Lehrkraft hat den Vorteil, dass etwaige Lesehürden, die es beim selbstständigen Durchlesen aufgrund von Entschlüsselungsschwierigkeiten geben könnte, nicht auftreten.

Im zweiten Rezeptionsdurchgang liegen keine geschlechtsspezifischen Präferenzen vor. Dass bei den Jungen die Präferenz für das Reden im Gegensatz zum Herausschreiben von Wörtern ausgeprägter ist als bei den Mädchen, mag an dem Sozialverhalten von Jungen liegen. Aus 
ihrem verbal dominierenden Verhalten, das oft eher als Ausdruck mangelnder Disziplin denn als Ausdruck thematischen Interesses beklagt wird ${ }^{46}$, scheinen die Jungen doch auch Nutzen für die schulischen Anforderungen zu ziehen.

3. In der Realschule liegen weniger geschlechtsspezifische Präferenzen als in der Hauptschule vor. Im ersten Rezeptionsdurchgang präferieren Mädchen signifikant das stille Lesen, Jungen etwa gleichrangig stilles Lesen und Aktivieren von Vorwissen.

Die Mädchen zeigen im ersten Rezeptionsdurchgang klare Präferenzen für das stille Durchlesen eines Textes. Sie erzielen damit ihr signifikant bestes Ergebnis und schneiden auch deutlich besser als die Jungen ab. Die am wenigsten geeignete Methode ist bei beiden Geschlechtern das Vorlesen eines Sachtextes. Dies mag daran liegen, dass für diese Textsorte das Vorlesen generell ungeeignet ist.

Im zweiten Rezeptionsdurchgang gibt es eine genderspezifische Besonderheit: Jungen schneiden mit Schreiben signifikant schlecht ab. Ansonsten erzielen Mädchen und Jungen mit allen Verfahren gleiche Ergebnisse.

Bei den Mädchen schneiden alle Verfahren gleich ab. Es ist gleichgültig, ob sie über den Text ein Unterrichtsgespräch führen oder ob sie Wörter unterstreichen bzw. herausschreiben. Jungen erzielen mit Reden über den Text und mit Unterstreichen die gleichen Leseverstehenserfolge wie die Mädchen. Doch zeigt sich deutlich der Misserfolg des Leseverstehens, wenn aus dem Sachtext Wörter herauszuschreiben sind.

Dass im Gegensatz zu der Hauptschule die Rangfolge der Strategien im zweiten Rezeptionsdurchgang bei den Mädchen völlig und bei den Jungen teilweise aufgehoben ist, lässt folgenden Schluss zu: Ab einem bestimmten Grad an Lesekompetenz ist das angewandte Verfahren bei der vertieften Textverarbeitung für den Leseverstehenserfolg unerheblich. Ausschlaggebend scheint eher die Art des Zugriffs auf den Sachtext bei der ersten Textbegegnung zu sein. Das signifikant schlechte Abschneiden der Jungen beim Schreiben lässt vermuten, dass die Jungen in der 7. Klasse Realschule erst auf dem Weg zur sicheren Anwendung schriftorientierter Verfahren sind.

4. Betrachtet man die Gesamtpopulation der Studie, also alle etwa 13-jährigen Mädchen und Jungen in der 7. Klasse (Hauptschule, Realschule), kommt man zu folgendem Ergebnis: Innerhalb der Gruppe der Mädchen ist eine größere Methodenvarianz zu verzeichnen als bei den Jungen. Hauptschülerinnen ziehen andere Verfahren vor als Realschülerinnen. Bei den Jungen gibt es weniger Unterschiede in der Methodenpräferenz. Auffallend ist das signifikant schlechte Abschneiden des Herausschreibens von Wörtern bei allen Jungen.

${ }^{46}$ Z.B. Rüegg (1995), Fuchs (2001), Kotthoff (2003). 
Bei den Mädchen zeigt sich ein deutlicher Unterschied zwischen Hauptschule und Realschule. Hauptschülerinnen schneiden mit Aktivieren von Vorwissen im ersten Rezeptionsdurchgang am besten ab, Realschülerinnen mit stillem Lesen. Bei beiden sind diese Präferenzen signifikant. Das Stilllesen führt bei den Hauptschülerinnen sogar zum schlechtesten Ergebnis.

Während es bei den Realschülerinnen im zweiten Rezeptionsdurchgang völlig gleichgültig ist, welches Verfahren im zweiten Rezeptionsdurchgang angewandt wird, zeigen die Hauptschülerinnen deutliche Präferenzen. Sie erzielen mit dem Reden über den Text ihre deutlich besten Ergebnisse. Herausschreiben von Wörtern führt zu den schlechtesten Ergebnissen. Im Kontext mit den generell schlechten Leseleistungen an Hauptschulen lässt die Präferenz für das Unterrichtsgespräch auf eine mangelnde Lesekompetenz bei Hauptschülerinnen schließen. Vermutlich können Hauptschülerinnen einen Sachtext nicht selbstständig lesen. Sie sind bei der Entnahme von Informationen auf die Lehrkraft angewiesen.

Bei den Jungen sind die Unterschiede in den Rezeptionsstrategien zwischen Haupt- und Realschule geringer. Jungen in der 7. Klasse sind in Bezug auf präferierte Rezeptionsstrategien daher eher als homogene Gruppe zu sehen. Den Jungen beider Schultypen ist eine gewisse Präferenz für das Stilllesen gemein. ${ }^{47}$ Im zweiten Rezeptionsdurchgang schneiden Haupt- und Realschüler mit Reden über den Text gut ab. Dieses Verfahren führt bei den Hauptschülern zu den besten Ergebnissen. Bei den Realschülern ist dies ein erfolgreiches Verfahren, das in den Ergebnissen jedoch mit dem Unterstreichen vergleichbar ist. Eine eindeutige Gemeinsamkeit haben Jungen beim Schreiben: Es schneidet bei beiden deutlich am schlechtesten ab.

Am Schreiben zeigt sich, dass die Präferenz von Rezeptionsstrategien nur bedingt geschlechtsspezifisch determiniert ist. Eine geschlechtsspezifische Differenz zeigt sich an der Realschule. Hier ziehen Mädchen aus dem Herausschreiben von Wörtern mehr Nutzen als Jungen. Vermutlich lassen sich Mädchen eher auf schulische Anforderungen ein, d.h. sie sind fleißiger und kommen durch Üben zu entsprechenden Ergebnissen. Nicht zu unterschätzen ist auch, dass Mädchen durch Tagebuchschreiben im Schreiben mehr geübt sind als Jungen. Mädchen befinden sich also früher als Jungen auf dem Weg zur Anwendung schriftorientierter Rezeptionsstrategien. Dass Rezeptionsstrategien auch bildungsbedingt sind, darauf verweisen die Ergebnisse in der Hauptschule. Mädchen wie Jungen haben mit dieser Rezeptionsstrategie gleichermaßen Schwierigkeiten. Vermutlich liegen die Defizite im schriftsprachlichen Bereich, die in der Hauptschule auch beim Unterstreichen von Schlüsselwörtern sichtbar sind, an der geringen Orientierung der Elternhäuser an Schriftlichkeit. Der Zusammenhang zwischen Herkunft, Schultyp und Lesekompetenz, auf den die PISA-Studien hingewiesen haben, wird auch hier augenfällig.

5. Beim Vergleich der Ergebnisse der vorliegenden Studie in der Sekundarstufe 7. Klasse mit den Ergebnissen in der Grundschul-Studie 4. Klasse gibt es deutliche Kontinuitäten:

Bei den Mädchen fand man in der Grundschul-Studie zwei Lesertypen vor: Mädchen, die eher mündliche bzw. kommunikationsorientierte Rezeptionsstrategien bevorzugen (Aktivieren

\footnotetext{
${ }^{47}$ Allerdings präferieren beide neben dem Stilllesen noch eine andere Methode: Hauptschüler das Vorlesen, Realschüler das Aktivieren von Vorwissen.
} 
von Vorwissen und Reden) und Mädchen, die eher schriftorientierte Rezeptionsstrategien (Stilllesen und Unterstreichen/Schreiben) bevorzugen. Es hat sich in der 7. Klasse herausgestellt, dass dieser Dualismus weiterhin besteht, die Präferenz für Rezeptionsstrategien jedoch vom Bildungsniveau abhängt.

Hauptschülerinnen kommen bei mündlichen Rezeptionsstrategien zu besseren Ergebnissen, Realschülerinnen erzielen mit dem Stilllesen eines Sachtextes ihre besten Ergebnisse. Eine Besonderheit bei Realschülerinnen ist, dass sie bei den Verfahren des zweiten Rezeptionsdurchgangs keinerlei Präferenzen aufweisen. Bei Leserinnen mit einer höheren Lesekompetenz ist vermutlich allein das Verfahren der ersten Textbegegnung ausschlaggebend.

Bei den Jungen führte in der Grundschul-Studie das Stilllesen eines Textes eindeutig zu den besten Ergebnissen. Dieser Befund liegt in abgeschwächter Form auch bei den Jungen in der 7. Klasse vor.

Jungen in der Sekundarstufe kommen mit dem stillen Durchlesen eines Textes und mit einer weiteren Methode zu guten Ergebnissen. Hauptschüler schneiden auch gut ab, wenn der Text vorgelesen wird, und Realschüler, wenn sie vor dem Lesen Vorwissen aktivieren.

Bei den Verfahren des zweiten Rezeptionsdurchgangs wurden bei den Jungen in der Sekundarstufe I deutliche Präferenzen offenbar, die so in der Grundschule nicht vorlagen. Das Schreiben, das in der Grundschule bei beiden Geschlechtern divergente Ergebnisse aufwies, führt bei Haupt- und Realschülern zum signifikant schlechtesten Ergebnis. Dieser Zusammenhang legt die Vermutung nahe, dass Jungen im Laufe der Schulzeit eine Abneigung gegen das Schreiben erwerben. Womöglich tritt ab einem bestimmten Alter ein "Schreibknick" ein - in Analogie zu dem verschiedentlich diagnostizierten "Leseknick" (Harmgarth 1999/Richter und Plath 2001/Bischoff/Heidtmann 2002b).

Die Autoren dieser Studie hoffen, mit den Ergebnissen aus der Leseforschung für die Didaktik des Lesens von Sachtexten einen Beitrag geleistet und den Lehrkräften Hinweise für die Konzipierung eines geschlechtsspezifisch differenzierten Unterrichts gegeben zu haben. In der Auswertungsphase befindet sich eine Parallelstudie im Gymnasium 7. Klasse (Grütz/Zmaila 2006). Wir würden uns über Rückmeldungen zu den Ergebnissen der Studie und zu Ihren eigenen Erfahrungen freuen. Bitte wenden Sie sich per E-mail an die Autorin dieses Beitrags: gruetz@ph-weingarten.de.

Ein Dank geht an die Gleichstellungsbeauftragte der Pädagogischen Hochschule Weingarten, Frau Prof. Dr. Gasteiger-Klicpera, die mit Mitteln des Gender-Etats die Auswertung der Daten dieser Studie unterstützt hat.

\section{Literaturangaben}

Alphen, Ingrid C. van (1996): "Wie die Jungen das Lachen lernten - und wie es den Mädchen wieder verging...." In: Kotthoff, Helga (ed.): Das Gelächter der Geschlechter. Konstanz: 217-227. 
Artelt, Cordula (2004): "Zur Bedeutung von Rezeptionsstrategien beim Textverstehen". In: Köster, Juliane et al. (eds): Aufgabenkultur und Lesekompetenz. Deutschdidaktische Positionen. Frankfurt, Berlin, Bern, Bruxelles, New York/Oxford/Wien.

Artelt, Cordula/Stanat, Petra/Schneider, Wolfgang/Schiefele, Ulrich (2001): "Lesekompetenz: Testkonzeption und Ergebnisse". In: Baumert et al.(eds.) (2001): PISA 2000. Basiskompetenzen von Schülerinnen und Schülern im internationalen Vergleich. Opladen: 69-137.

Baumert, Jürgen/Klieme, Eckhard/Neubrand, Michael/Prenzel, Manfred/Schiefele, Ulrich/Schneider, Wolfgang/Stanat, Petra/Tillmann, Klaus-Jürgen/Weiß, Manfred (eds.) (2001): PISA 2000. Basiskompetenzen von Schülerinnen und Schülern im internationalen Vergleich. Opladen.

Baumert, Jürgen/Artelt, Cordula/Klieme, Eckhard/Neubrand, Michael/Prenzel, Manfred/Schiefele, Ulrich/Schneider, Wolfgang/Tillmann, Klaus-Jürgen/Weiß, Manfred (eds.) (2003): PISA 2000 - Ein differenzierter Blick auf die Länder der Bundesrepublik Deutschland. Opladen.

Baron-Cohen, Simon (2004): Vom ersten Tag an anders. Das weibliche und das männliche Gehirn. Psychologie heute März 2004: 44-47.

Belgrad, Jürgen/Grütz, Doris/Pfaff, Harald (2003): Forschungsbericht 2003. Studie zum Verstehen von Sachtexten in der Grundschule 4. Klasse. Pädagogische Hochschule Weingarten www.ph-weingarten.de/deutsch.

Belgrad, Jürgen/Grütz, Doris/Pfaff, Harald (2004): "Verstehen von Sachtexten. Eine Studie in der Grundschule 4. Klasse". Didaktik Deutsch 17: 26-43.

Bertschi-Kaufmann, Andrea (2002a): "Multimedia und Leseförderung in der Schule. Ergebnisse aus den Forschungsprojekten 'Literalität im medialen Umfeld' und 'Lesen im Kontext neuer Medien'". In: Bonfadelli, Heinz/Bucher, Priska (eds.): Lesen in der Mediengesellschaft. Stand und Perspektive der Forschung. Zürich: 148-161.

Bertschi-Kaufmann, Andrea (2002b): "Lesen und Schreiben in einer Medienumgebung. Empirisches Arbeiten im Raum Schule". In: Kammler, Clemens/Knapp, Werner (eds.). Empirische Unterrichtsforschung und Deutschdidaktik. Reihe Diskussionsforum Deutsch. Bd.5. Baltmannsweiler: 141-155.

Bischoff, Ulrike/Heidtmann, Horst (2002a): Leseverhalten in der Erlebnisgesellschaft. Eine Untersuchung zu den Leseinteressen und Lektüregratifikationen von Jungen. Weinheim, München: $241-267$.

Bischoff, Ulrike/Heidtmann, Horst (2002b): "Lesen Jungen ander(e)s als Mädchen? Untersuchungen zu Leseinteresse und Lektüregratifikationen". medien praktisch. Zs. für Medienpädagogik Heft 3/02. Nr. 103: 27-31.

Bonfadelli, Heinz/Bucher, Priska (eds.) (2002): Lesen in der Mediengesellschaft. Stand und Perspektive der Forschung. Zürich.

Bonfadelli, Heinz/Fritz, Angela (1993): "Lesen im Alltag von Jugendlichen". In: Bonfadelli, Heinz/Fritz, Angela/Köcher, Renate (1993): Lesesozialisation. Bd.2. Leseerfahrungen und Lesekarrieren: Studien der Bertelsmann Stiftung. Gütersloh: 7-213. 
Bonfadelli, Heinz/Fritz, Angela/Köcher, Renate (1993): Lesesozialisation. Bd.2. Leseerfahrungen und Lesekarrieren: Studien der Bertelsmann Stiftung. Gütersloh.

Bos, Wilfried/Lankes, Eva-Maria/Prenzel, Manfred/Schwippert, Kurt/Walther, Gerd/Valtin, Renate (eds.) (2003): Erste Ergebnisse aus IGLU. Schülerleistungen am Ende der vierten Jahrgangsstufe im internationalen Vergleich. Münster etc.

Brügelmann, Hans/Balhorn, Heiko/Füssenich, Iris (eds.) (1995): Am Rande der Schrift. Zwischen Sprachenvielfalt und Analphabetismus. Lengwil.

Christmann, Ursula/Groeben, Norbert (2002): "Anforderungen und Einflussfaktoren bei Sach- und Informationstexten". In: Groeben, Norbert/Hurrelmann, Bettina (eds.) (2002): Lesekompetenz - Bedingungen, Dimensionen, Funktionen. Weinheim und München: 150173.

Demmer, Corinna (2005): Leseverstehen und Interesse. Eine Untersuchung in der Hauptschule 7. Klasse. Wissenschaftliche Hausarbeit an der Pädagogischen Hochschule Weingarten. Weingarten.

Eggert, Hartmut/Garbe, Christine (1995): Literarische Sozialisation. Stuttgart/Weimar.

Elley, Warwick Barham (ed.) (1994): The IEA Study of Reading Literacy: Achievement and instruction in thirty-two school systems. Oxford.

Franz, Kurt/Franzmann, Bodo/Payrhuber, Franz-Josef/Schön, Erich (1999): "Muß-Lektüre versus Lust-Lektüre?" In: Groeben, Norbert (ed.): Lesesozialisation in der Mediengesellschaft. Internationales Archiv für Sozialgeschichte der deutschen Literatur. 10. Sonderheft. Tübingen: 78-88.

Fuchs, Claudia (2001): Barbie trifft He-Man. Kinder erzählen über ihre Spielwelten und ihre Alltagswelt. Freiburg i. Brsg.

Garbe, Christine (2003a): "Warum lesen Mädchen besser als Jungen? Zur Notwendigkeit einer geschlechterdifferenzierenden Leseforschung und Leseförderung". In: Abraham, Ulf et al.: Deutschdidaktik und Deutschunterricht nach PISA. Freiburg i. Brsg.: 69-89.

Garbe, Christine (2003b): "Mädchen lesen ander(e)s". JuLit 2/03: 14-29.

Groeben, Norbert (ed.) (1999): Lesesozialisation in der Mediengesellschaft. Internationales Archiv für Sozialgeschichte der deutschen Literatur. 10. Sonderheft. Tübingen.

Groeben, Norbert/Hurrelmann, Bettina (eds.) (2002): Lesekompetenz - Bedingungen, Dimensionen, Funktionen. Weinheim und München.

Grütz, Doris (2004): "Der geschlechtsspezifische Zugriff auf Rezeptionsstrategien Ergebnisse einer Untersuchung im Rahmen unterrichtsdidaktischer Forschung". Linguistik online 21, 4/04: 23-46. http://www.linguistik-online.de/21_04/gruetz.html.

Grütz, Doris/Belgrad, Jürgen/Pfaff, Harald (2004): Forschungsbericht Sekundarstufe I. Studie zum Verstehen von Sachtexten in der Hauptschule und der Realschule 7. Klasse. Pädagogische Hochschule Weingarten.

http://www.ph-weingarten.de/ > Lehre und Forschung > Fakultät II > Deutsch > Homepage Deutsch.

Hamm, Ingrid/Langen, Claudia (2002): Das Lesebarometer - Lesen und Umgang mit Büchern in Deutschland. In: Bonfadelli, Heinz/Bucher, Priska (eds.): Lesen in der Mediengesellschaft. Stand und Perspektive der Forschung. Zürich: Pestalozzianum: 43-56. 
Harmgarth, Friederike (ed.) (1999): Das Lesebarometer - Lesen und Umgang mit Büchern in Deutschland. Eine Bestandsaufnahme zum Leseverhalten von Kindern und Erwachsenen. Gütersloh.

Hurrelmann, Bettina (2002): "Prototypische Merkmale der Lesekompetenz". In: Groeben, Norbert/Hurrelmann, Bettina (eds.): Lesekompetenz - Bedingungen, Dimensionen, Funktionen. Weinheim und München: 275-286.

Hurrelmann, Bettina (2003): "Leseleistung - Lesekompetenz. Folgerungen aus PISA, mit einem Plädoyer für ein didaktisches Konzept des Lesens als kultureller Praxis". In: Menzel, Wolfgang (ed.): Texte lesen - Texte verstehen. Praxis Deutsch. Sonderheft. Seelze: $10-21$.

Hurrelmann, Bettina/Hammer, Michael /Nieß, Ferdinand (1993): Lesesozialisation. Bd.1. Leseklima in der Familie: eine Studie der Bertelsmann Stiftung. Gütersloh.

Kammler, Clemens/Knapp, Werner (eds.) (2002): Empirische Unterrichtsforschung und Deutschdidaktik. Baltmannsweiler: Schneider (= Reihe Diskussionsforum Deutsch Bd.5).

Kessels, Ursula (2004): "Mädchenfächer - Jungenfächer? Geschlechtertrennung im Unterricht". In: Becker, Gerold et al. (eds.): Heterogenität. Unterschiede nutzen Gemeinsamkeiten stärken. Friedrich Jahresheft XXII 2004: 90-94.

Kintsch, Walter (1996): "Lernen aus Texten". In: Hoffmann, Joachim/Kintsch, Walter (eds.): Lernen. Enzyklopädie der Psychologie. Themenbereich C, Serie II, Band 7. Göttingen: 503-528.

Köcher, Renate (1993): "Lesekarrieren - Kontinuität und Brüche". In: Bonfadelli, Heinz/Fritz, Angela/Köcher, Renate (1993): Lesesozialisation. Bd.2. Leseerfahrungen und Lesekarrieren: Studien der Bertelsmann Stiftung. Gütersloh: 215-310.

Köster, Juliane (2003): "Die Bedeutung des Vorwissens in der Lesekompetenz". In: Abraham, Ulf et al.: Deutschdidaktik und Deutschunterricht nach PISA. Freiburg i. Brsg.: 90-105.

Kotthoff, Helga (2003): "Problemgruppe Jungen? Neue Fragen für die Kinder- und Jugendforschung, Ethnografie der Schule und die Gesprächsanalyse". Der Deutschunterricht 2/2003: 85-88.

Kübler, Hans-Dieter (1999): "Mann und Buch - ein Widerspruch? Erkenntnisse aus der Leseforschung über ein weithin unbeachtetes Thema". Buch und Bibliothek 7/8: 468-475.

Lehmann, Rainer H. (1994). "Lesen Mädchen wirklich besser? Ergebnisse aus der internationalen IEA-Lesestudie". In: Richter, Sigrun/Brügelmann, Hans (eds.): Mädchen lernen ANDERS lernen Jungen: Geschlechtsspezifische Unterschiede beim Schriftspracherwerb. Konstanz und Bremen: 99-109.

Lehmann, Rainer H./Peek, Rainer/Poerschke, Jan (1997): HAMLET 3-4. Hamburger Lesetest für 3. und 4. Klassen. Weinheim und Basel.

Linke, Angelika/Oomen-Welke, Ingelore (eds.): Herkunft, Geschlecht und Deutschunterricht: oben - unten, von hier - von anderswo, männlich - weiblich. Freiburg i. Brsg.

Naceur, Abdelmajid (2001): Interesse und Textverstehen. Dissertation. Bielefeld.

Nickel-Bacon, Irmgard (2003): "Hat Lesen ein Geschlecht?" Schüler 2003. Lesen + Schreiben: $30-31$.

Oakhill, Jane/Garnham, Alan (1988): Becoming a skilled reader. New York. 
Oerter, Rolf (1999): "Theorien der Lesesozialisation - Zur Ontogenese des Lesens". In: Groeben, Norbert (ed.): Lesesozialisation in der Mediengesellschaft. Internationales Archiv für Sozialgeschichte der deutschen Literatur. 10. Sonderheft. Tübingen: 27-55.

Paris, Scott G./Oka, Evelyn R. (1986): "Children's reading strategies, metacognition, and motivation". Developmental Review 6: 25-26.

Perfetti, Charles A. (1985): Reading ability. New York.

Pressley, Michael et al. (1985): "Children's use of cognitive strategies, how to teach strategies, and what to do if they can't be taught". In: Pressley, Michael/Brainerd, Charles J. (ed.): Cognitive learning and memory in children. New York: 1-47.

Richter, Karin/Plath, Monika (2002): "Die Bedeutung der Entwicklung von Lesemotivation in der Grundschule". In: Franz, Kurt/Payrhuber, Franz-Josef (eds): Lesen heute. Leseverhalten und Leseförderung im Kontext der PISA-Studie. Baltmannsweiler: 41-58.

Richter, Sigrun: "Mädchen und Jungeninteressen beim Schreiben und Lesen". Grundschule 12/1999: 38-40.

Richter, Sigrun/Brügelmann, Hans (eds.) (1994): Mädchen lernen anders - anders lernen Jungen: Geschlechtsspezifische Unterschiede beim Schriftspracherwerb. Lengwil.

Richter, Tobias/Christmann, Ursula (2002): "Lesekompetenz: Prozessebenen und interindividuelle Unterschiede". In: Groeben, Norbert/Hurrelmann, Bettina (eds.) (2002): Lesekompetenz - Bedingungen, Dimensionen, Funktionen. Weinheim und München: 2558.

Rüegg, Regula (1995): "Stille Mädchen - laute Buben?" In: Linke, Angelika/Oomen-Welke, Ingelore: Herkunft, Geschlecht und Deutschunterricht: oben - unten, von hier - von anderswo, männlich - weiblich. Freiburg i. Brsg.: 171-188.

Rumelhart, David E. (1975): "Notes on a schema for stories". In: Bobrow, Daniel G./Collins, Allan (eds.): Representation and understanding. New York.

Rumelhart, David E. (1994): "Toward an interactive model of reading". In: Singer, Harry (ed.): Theoretical models and processes of reading. Newark Delaware: International Reading Association: 864-894.

Schilcher, Anita (2003): "Geschlechterdifferenzierender Deutschunterricht. Was machen die Jungs? Geschlechterdifferenzierender Deutschunterricht nach PISA". In: Abraham, Ulf et al.: Deutschdidaktik und Deutschunterricht nach PISA. Freiburg i. Brsg.: 361-380.

Spiro, Rand J. (1980): "Constructive processes in prose comprehension and recall". In: Spiro, Rand J./Bruce, Bertram C./Brewer, William F. (eds.): Theoretical issues in reading comprehension: Perspectives from cognitive psychology, linguistics, artificial intelligence and education. Hillsdale: 245-278.

Stanat, Petra/Kunter, Mareike (2001): "Geschlechterunterschiede in Basiskompetenzen". In: Baumert, Jürgen/Klieme, Eckhard/Neubrand, Michael/Prenzel, Manfred/Schiefele, Ulrich/Schneider, Wolfgang/Stanat, Petra/Tillmann, Klaus-Jürgen/Weiß, Manfred (eds.) (2001): PISA 2000. Basiskompetenzen von Schülerinnen und Schülern im internationalen Vergleich. Opladen: 249-269. 
Stanat, Petra et al. (2003): "PISA und PISA-E: Zusammenfassung der bereits vorliegenden Befunde". In: Deutsches PISA-Konsortium (eds.): PISA 2000. Ein differenzierter Blick auf die Länder der Bundesrepublik Deutschland. Opladen: 51-75.

Stanovich, Keith E.(1980): "Toward an interactive-compensatory model of individual differences in the developpement of reading fluency". Reading Research Quaterly 16: 3271.

Stiftung Lesen (eds.) (2001): Leseverhalten in Deutschland im neuen Jahrtausend. Schriftenreihe "Lesewelten". Bd. 3. Mainz.

Thimm, Katja (2004): "Angeknackste Helden". Der Spiegel 21/2004: 82-95.

Van Dijk, Teun A./Kintsch, Walter (1983): Strategies of discourse comprehension. New York.

Van Kraayenoord, Christina E./Schneider, Wolfgang (1999): "Reading achievement, metacognition, reading self-concept and interest. A study of german students in grade 3 and 4". European Journal of psychology and Education 14: 305-324.

Yekovich, Frank R./Walker, Carol H./Blackmann, Harold S. (1979): "The role of presupposed and focal information in integrating sentences". Journal of Verbal Learning and Verbal Behaviour 18.

\section{Anhang}

Teilnehmer/innen an der Studie

\begin{tabular}{|c|c|c|c|c|c|c|}
\hline \multirow{2}{*}{$\begin{array}{l}\text { Sekundarstufe I } \\
\text { Klasse } 7 \\
\end{array}$} & \multicolumn{2}{|c|}{ Mädchen } & \multicolumn{2}{|c|}{ Jungen } & \multicolumn{2}{|c|}{ gesamt } \\
\hline & absolut & $\%$ & absolut & $\%$ & absolut & $\%$ \\
\hline Hauptschule & 713 & $41,6 \%$ & 998 & $58,2 \%$ & 1716 & Insgesamt \\
\hline Realschule & 1333 & $51,3 \%$ & 1245 & $48,1 \%$ & 2586 & $0,3 \%$ \\
\hline alle & 2046 & $47,6 \%$ & 2243 & $52,1 \%$ & 4302 & \\
\hline
\end{tabular}

Tab. 1: Zahl der an der Studie teilgenommenen Mädchen und Jungen 

bei Mädchen und Jungen in der Sekundarstufe I

\begin{tabular}{|c|c|c|}
\hline Mädchen & Hauptschule & Realschule \\
\hline $\begin{array}{l}\text { Beste } \\
\text { Methoden- } \\
\text { kombination }\end{array}$ & $\begin{array}{l}\text { M8 Aktivierung von Vorwissen + } \\
\text { Reden } \\
\text { M7 Aktivierung von Vorwissen }+ \\
\text { Unterstreichen }\end{array}$ & $\begin{array}{l}\text { M3 Stilllesen }+ \text { Schreiben } \\
\text { M2 Stilllesen }+ \text { Reden } \\
\text { M7 Aktivierung von Vorwissen }+ \\
\quad \text { Unterstreichen }\end{array}$ \\
\hline $\begin{array}{l}\text { Schlechteste } \\
\text { Methoden- } \\
\text { kombination }\end{array}$ & $\begin{array}{l}\text { M9 Aktivierung von Vorwissen + } \\
\text { Schreiben } \\
\text { M1 Stilllesen + Unterstreichen }\end{array}$ & M6 Vorlesen und Schreiben \\
\hline $\begin{array}{c}\text { 1. Rezeptions- } \\
\text { durchgang }\end{array}$ & $\begin{array}{l}\text { 1. Aktivieren von Vorwissen } \\
\quad \text { (Signifikant am besten) } \\
\text { 2. Vorlesen } \geq 3 \text {. Stilllesen } \\
\text { - Aktivieren von Vorwissen }>\text { Vorlesen } 0,04 * \\
\text { - Aktivieren von Vorwissen }>\text { Stilllesen } 0,01 * \\
\text { - Vorlesen - Stillesen n. s. }\end{array}$ & $\begin{array}{l}\text { 1. Stilllesen (signifikant am besten) } \\
\text { 2. Aktivieren von Vorwissen } \\
\text { 3. Vorlesen } \\
\text { - Stilllesen }>\text { Aktivieren von } \\
\text { Vorwissen } 0,01^{*} \\
\text { - Stillesen }>\text { Vorlesen } 0,00 * * \\
\text { - Aktivieren von Vorwissen }>\text { Vorlesen } \\
\text { n. s. }\end{array}$ \\
\hline $\begin{array}{l}\text { 2. Rezeptions- } \\
\text { durchgang }\end{array}$ & $\begin{array}{l}\text { 1. Reden } \\
\text { 2. Unterstreichen } \\
\text { 3. Schreiben } \\
\text { - Reden }>\text { Unterstreichen } 0,17 \text { n.s. } \\
\text { - Reden }>\text { Schreiben } 0,03 * \\
\text { - Unterstreichen }>\text { Schreiben } 0,38 \text { n.s. }\end{array}$ & $\begin{array}{l}\text { keine signifikanten Unterschiede } \\
\text { (fast gleiche Mittelwerte) }\end{array}$ \\
\hline
\end{tabular}

Tab. 2: Unterschiede zwischen Mädchen an der Hauptschule und an der Realschule Klasse 7

n. s.: nicht signifikant $\quad *$ : signifikant $(\mathrm{p} \leq 0,05) \quad * *$ : hochsignifikant $(\mathrm{p} \leq 0,00)$ 


\begin{tabular}{|c|c|c|}
\hline Jungen & Hauptschule & Realschule \\
\hline $\begin{array}{l}\text { Beste } \\
\text { Methoden- } \\
\text { kombination }\end{array}$ & $\begin{array}{l}\text { alle Methodenkombinationen mit dem } \\
\text { Reden über den Text } \\
\text { als } 2 \text {. Rezeptionsdurchgang }\end{array}$ & $\begin{array}{l}\text { M7 Aktivierung von Vorwissen + } \\
\quad \text { Unterstreichen }\end{array}$ \\
\hline $\begin{array}{l}\text { Schlechteste } \\
\text { Methoden- } \\
\text { kombination }\end{array}$ & $\begin{array}{l}\text { M9 Aktivierung von Vorwissen + } \\
\quad \text { Schreiben }\end{array}$ & M6 Vorlesen + Schreiben \\
\hline $\begin{array}{l}\text { 1. Rezeptions- } \\
\text { durchgang }\end{array}$ & $\begin{array}{l}\text { Keine signifikanten Unterschiede; } \\
\text { aber Tendenz der Jungen zum } \\
\text { Vorlesen und zum Stilllesen; } \\
\text { hierbei gegenüber den Mädchen } \\
\text { signifikant bessere Leseleistungen }\end{array}$ & $\begin{array}{l}\text { keine signifikanten Unterschiede } \\
\text { Aktivieren von Vorwissen entspricht } \\
\text { Stilllesen } \\
\text { Aktivieren von Vorwissen > Vorlesen fast * }\end{array}$ \\
\hline $\begin{array}{l}\text { 2. Rezeptions- } \\
\text { durchgang }\end{array}$ & $\begin{array}{l}\text { 1. Reden } \\
\text { 2. Unterstreichen } \\
\text { 3. Schreiben } \\
\text { Reden }>\text { Unterstreichen * } \\
\text { Reden }>\text { Schreiben } * * \\
\text { Unterstreichen }>\text { Schreiben * }\end{array}$ & $\begin{array}{l}\text { 1. Reden / Unterstreichen } \\
\text { 3. Schreiben } \\
\text { Reden entspricht Unterstreichen } \\
\text { Reden }>\text { Schreiben * } \\
\text { Unterstreichen }>\text { Schreiben fast* } \\
\rightarrow \text { Schreiben signifikant schlechter }\end{array}$ \\
\hline
\end{tabular}

Tab. 3: Unterschiede zwischen Jungen an der Hauptschule und an der Realschule Klasse 7

n. s.: nicht signifikant $\quad *$ : signifikant $(\mathrm{p} \leq 0,05) \quad * *$ : hochsignifikant $(\mathrm{p} \leq 0,00)$ 

bei Mädchen und Jungen in der Sekundarstufe I

\begin{tabular}{|c|c|c|}
\hline $\begin{array}{l}\text { Unterschiede } \\
\text { Mädchen - Jungen }\end{array}$ & Hauptschule & Realschule \\
\hline Methodenkombinationen & $\begin{array}{l}\text { Insgesamt keine } \\
\text { signifikanten Unterschiede: } \\
\text { Mädchen: 2,66 - Jungen: 2,71 } \\
\text { Jungen besser als Mädchen: } \\
\text { M5 Vorlesen + Reden* }\end{array}$ & $\begin{array}{l}\text { Insgesamt keine } \\
\text { signifikanten Unterschiede: } \\
\text { Mädchen: 3,62 - Jungen: 3,60 } \\
\text { Mädchen besser als Jungen: } \\
\text { M3 Still lesen + Schreiben* }\end{array}$ \\
\hline 1. Rezeptionsdurchgang & $\begin{array}{l}\text { Jungen signifikant besser bei } \\
\text { Stillesen* und Vorlesen* } \\
\text { Jungen besser bei allen } \\
\text { Methodenkombinationen mit } \\
\text { Stilllesen und Vorlesen; } \\
\text { außer: M6 Vorlesen + Schreiben } \\
\text { (hier gleiches Ergebnis) } \\
\text { Mädchen (fast signifikant besser) } \\
\text { bei allen Methodenkombinationen } \\
\text { mit Aktivieren von Vorwissen } \\
\text { (p: } 0,07 \text { ) }\end{array}$ & $\begin{array}{l}\text { Stilllesen fast signifikant besser } \\
\text { zugunsten Mädchen }(0,06) \\
\text { Sonst keine signifikanten } \\
\text { Unterschiede }\end{array}$ \\
\hline 2. Rezeptionsdurchgang & keine signifikanten Unterschiede & keine signifikanten Unterschiede \\
\hline
\end{tabular}

Tab. 4: Geschlechtsspezifische Unterschiede bei Hauptschulen und Realschulen Klasse 7 im Vergleich
n. s.: nicht signifikant
*: signifikant $(\mathrm{p} \leq 0,05)$
$* *$ : hochsignifikant $(\mathrm{p} \leq 0,00)$ 


\begin{tabular}{|c|c|c|c|c|}
\hline \multicolumn{2}{|c|}{ Hauptschule - Methodenkombinationen } & \multirow{2}{*}{$\begin{array}{c}\text { weiblich } \\
2,42 \\
\end{array}$} & \multirow{2}{*}{$\begin{array}{c}\text { männlich } \\
2,66 \\
\end{array}$} & \multirow{2}{*}{$\begin{array}{c}\mathbf{p} \\
0,14 \text { (n.s.) }\end{array}$} \\
\hline M1 & Stilllesen + Unterstreichen & & & \\
\hline M2 & Stilllesen + Reden & 2,60 & 3,00 & $0,14(\text { n. s. })^{48}$ \\
\hline M3 & Stilllesen + Schreiben & 2,57 & 2,70 & 0,50 ( n. s.) \\
\hline M4 & Vorlesen + Unterstreichen & 2,59 & 2,74 & 0,33 (n.s.) \\
\hline M5 & Vorlesen + Reden & 2,54 & 2,92 & $\mathbf{0 , 0 3} *$ \\
\hline M6 & Vorlesen + Schreiben & 2,65 & 2,67 & 0,90 ( n. s.) \\
\hline M7 & Aktivierung von Vorwissen + Unterstreichen & 2,90 & 2,77 & 0,42 ( n. s.) \\
\hline M8 & Aktivierung von Vorwissen + Reden & 3,15 & 2,94 & 0,22 ( n.s.) \\
\hline M9 & Aktivierung von Vorwissen + Schreiben & 2,49 & 2,27 & 0,16 (n.s.) \\
\hline & Gesamt & 2,66 & 2,71 & $\mathbf{0 , 3 3}$ (n. s.) \\
\hline
\end{tabular}

Tab. 5: Mittelwerte nach Geschlecht in den Hauptschulen

n. s.: nicht signifikant $\quad *$ : signifikant $(\mathrm{p} \leq 0,05)$

${ }^{48}$ Hier liegt wegen der zu geringen Zellenbesetzung kein signifikanter Unterschied vor. 

bei Mädchen und Jungen in der Sekundarstufe I

\begin{tabular}{|c|c|c|c|c|}
\hline \multicolumn{2}{|c|}{ Realschule - Methodenkombinationen } & weiblich & männlich & $\mathbf{P}$ \\
\hline M1 & Stilllesen + Unterstreichen & 3,54 & 3,54 & 0,99 (n.s.) \\
\hline M2 & Stilllesen + Reden & 3,83 & 3,77 & 0,66 (n.s.) \\
\hline M3 & Stilllesen + Schreiben & 3,89 & 3,61 & $0,04 *$ \\
\hline M4 & Vorlesen + Unterstreichen & 3,57 & 3,64 & 0,57 (n.s.) \\
\hline M5 & Vorlesen + Reden & 3,59 & 3,78 & 0,20 (n. s.) \\
\hline M6 & Vorlesen + Schreiben & 3,11 & 3,18 & 0,64 (n.s.) \\
\hline M7 & Aktiv Vorwissen + Unterstreichen & 3,79 & 3,93 & 0,44 (n.s.) \\
\hline M8 & Aktiv Vorwissen + Reden & 3,47 & 3,50 & 0,83 (n.s.) \\
\hline M9 & Aktiv Vorwissen + Schreiben & 3,59 & 3,70 & 0,37 (n.s.) \\
\hline & Gesamt & 3,62 & 3,60 & 0,75 (n. s.) \\
\hline
\end{tabular}

Tab. 6: Mittelwerte der Methodenkombinationen nach Geschlecht an Realschulen n. s.: nicht signifikant $*$ : signifikant $(\mathrm{p} \leq 0,05)$ 


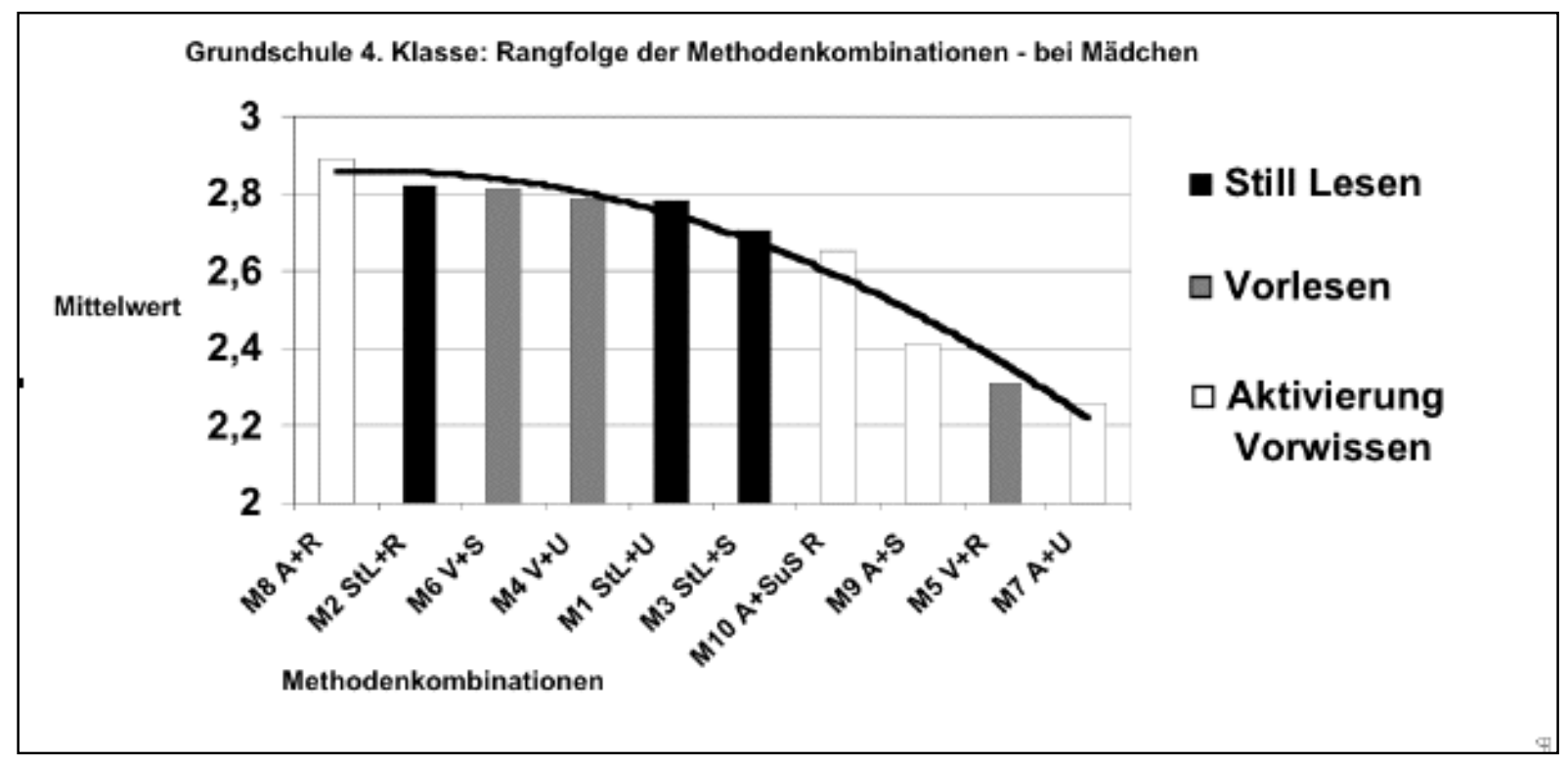

Tab. 7: $\quad$ Rangfolge der Methodenkombinationen bei Mädchen in der Grundschule 4. Klasse $^{49}$

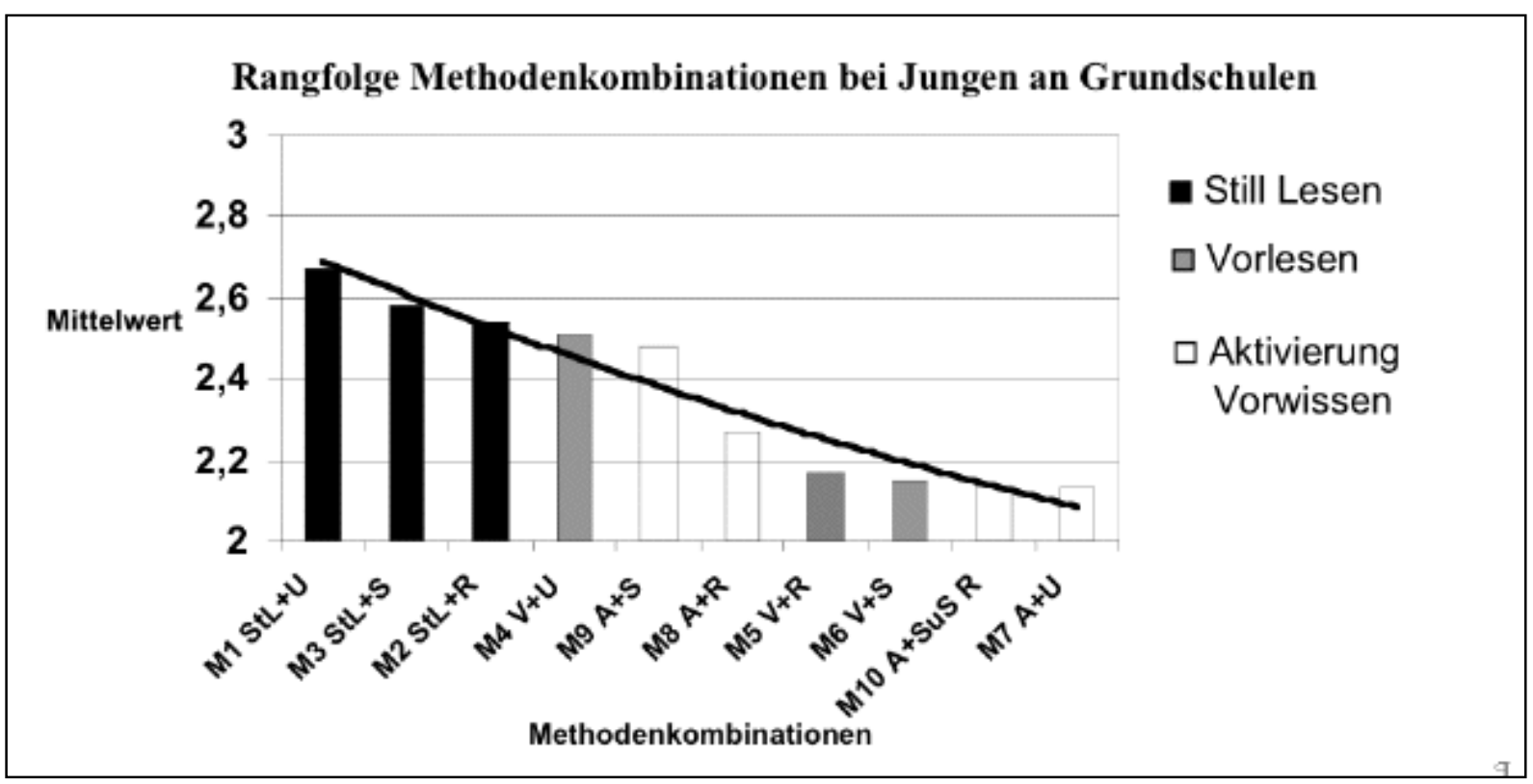

Tab. 8: $\quad$ Rangfolge der Methodenkombinationen bei Jungen in der Grundschule 4. Klasse

49 Die Tabellen 6 und 7 zur Grundschul-Studie (Belgrad/Grütz/Pfaff 2004) dürfen nur in Bezug auf ihre Rangfolge, nicht aber in Bezug auf ihre Skalierung bei der Leseleistung (Ordinate) verglichen werden. Im Test der Grundschul-Studie waren vier, im Test der Sekundarstufen-Studie 7. Klasse fünf Punkte zu erreichen. Zur Begründung s. Grütz, Belgrad \& Pfaff (2004): Forschungsbericht. Teil A Kap.2.4.

Die Methodenkombination M10 Aktivierung von Vorwissen und Schüler reden wurde in der Grundschul-Studie 4. Klasse zusätzlich zum Forschungsdesign mitgetestet. Die Wertung dieser Methodenkombination als Erfolg hatte sich vor allem darin begründet, dass das Verfahren "Schülerinnen und Schüler reden" in einer 4. Klasse noch nicht zu den eingeübten Verfahren gehört. 